\title{
Power generation portfolios: A parametric formulation of the efficient frontier
}

\author{
David Juárez-Luna1 - Universidad Anáhuac México, México
}

This paper aims to provide a methodology to construct parametrically the Efficient Frontier (EF) of Power Generation Portfolio (PGP). The methodology works as follows. First, we obtain two sets of the shares of the assets: one that guarantee the maximal expected return on the PGP; and another that guarantee the minimal risk of the PGP. The EF corresponds to the parametric equation of the risk-return profiles from the minimal risk to the maximal expected return of the PGP. We apply our methodology to replicate the results from three existing papers. The present methodology allows to and different and more coherent results than those obtained in the original papers. The analysis suggests that there are optimal investment alternatives that have been denied by previous analysis. This fact creates a bias in the design of investment policies in electricity generation. One limitation of the paper is that the analysis relies on the assumption that the covariances of the returns of the different assets is zero. This assumption leads to gains in tractability, clarity, and in the scope of the methodology formulated.

JEL Classification: D81, G11, Q40, Q49

Keywords: Portfolio, Power Generation, Efficient Frontier, Risk, Return.

\section{Carteras de generación de energía: Una formulación paramétrica de la frontera eficiente}

El objetivo de este artículo es proporcionar una metodología para construir, paramétricamente, la frontera eficiente (EF, por sus siglas en inglés) de portafolios de generación de energía (PGP, por sus siglas en inglés). La metodología opera de la siguiente manera. Primero, obtenemos dos conjuntos de las participaciones de los activos: uno que garantiza el máximo rendimiento del PGP; y otro que garantiza el riesgo mínimo del PGP. La EF corresponde a la ecuación paramétrica de los perfiles de riesgo-rendimiento, desde el riesgo mínimo hasta el máximo rendimiento esperado del PGP. La metodología propuesta se aplica para replicar los resultados de tres artículos existentes. La presente metodología permite encontrar resultados diferentes y más coherentes que los obtenidos en los documentos originales. El análisis sugiere que existen alternativas de inversión óptimas que han sido negadas por análisis previos. Este hecho crea un sesgo en el diseño de políticas de inversión en la generación de electricidad. Una limitante del trabajo es que el análisis se basa en el supuesto de que las covarianzas de los rendimientos de los diferentes activos son cero. Este supuesto implica ganancias en cuanto al manejo, la claridad y en el alcance de la metodología formulada.

Clasificación JEL: D81, G11, Q40, Q49

Palabras clave: Portafolio, generación de energía, frontera eficiente, riesgo, rendimiento.

\footnotetext{
${ }^{1}$ Corresponding author. Universidad Anáhuac México. Av. Universidad Anáhuac 46, Col. Lomas Anáhuac, Huixquilucan, Estado de México, México. C. P 52786. E-mail address: david.juarez@anahuac.mx.

* No source of funding for research development
} 


\section{Introduction}

Power generation is subject to different sources of uncertainty (e.g., changes in fuel and electricity prices, changes in the cost of $\mathrm{CO}_{2}$ emissions, and changes in the interest rate). Therefore, uncertainty exerts a direct impact on the risk-return profiles of the generation of different technologies. In this regard, the Portfolio Theory, developed by Markowitz (1952), has been extensively used to design plans for power generation that reduce risk and increase returns through diversification (see DeLlano-Paz et al. (2017), for a review). However, existing papers do not provide a description on how the authors construct the Efficient Frontier (EF) of the Power Generation Portfolio (PGP) analyzed (e.g., Perea González and Zavaleta Vazquez (2020), Gómez-Ríos and Juárez-Luna (2019), Costa et al. (2017), Pinheiro Neto et al. (2017), Adams and Jamasb (2016), Jain et al. (2014), Cunha and Ferreira (2014), Francés et al. (2013), Losekann et al. (2013), Arnesano et al. (2012), Bhattacharya and Kojima (2012) Allan et al. (2011), Delarue et al. (2011), Roques et al. (2010), Vithayasrichareon et al. (2010a), Vithayasrichareon et al. (2010b), Roques et al. (2008), Roques (2006), and Awerbuch and Berger (2003)). The construction of the EF, performed mostly by simulation, remains as a black box.

In the present paper, we aim to open such a black box by providing a detailed methodology for the parametric construction of the EF of PGP of up to five assets. ${ }^{2}$ Following the existing literature, in the present analysis the EF refers to the set of the PGP that maximize their expected returns for a given level of risk. That is, the expected return of an efficient PGP can be increased only by increasing its risk (Awerbuch and Berger (2003)). The present methodology could also be applied to PGP based on the Levelized Cost of Electricity (LCOE). In such a case, the EF would be a set of PGP that can yield the lowest expected LCOE at given levels of expected risk (Jansen et al. (2006)).

The analysis relies on the fact that the risk of the PGP is a convex function of the shares of the different assets. The methodology works as follows: first, obtain the shares of the assets that guarantee the maximal expected return on the PGP. Then, obtain the shares of the assets that guarantee the minimal risk of the PGP. We then formulate the risk of the efficient generation portfolios as a parametric equation of the shares of the assets. Finally, the EF corresponds to the parametric equation of the risk-return profiles from the minimal risk of the PGP to the maximal expected return of the PGP.

The majority of the existing literature on energy portfolios follows a two-step methodology proposed by Roques et al. (2008). In the first step, the distribution of the returns is simulated by applying Monte Carlo simulation or real options theory. In the second step, the portfolio theory is applied to simulated data to find the optimal generation portfolios. In this paper we focus on the second step of such a methodology.

To exemplify the advantages and the scope of the methodology, we apply it to replicating and extending the existing results from the following three papers: Roques et al. (2008), Jain et al. (2014), and Roques et al. (2010). Among the main results, we have the following:

Roques et al. (2008) analyze portfolios of three technologies: CyCle gas turbine (CCGT), coal, and the nuclear plant. These authors conduct the analysis with pairs of technologies under three scenarios as follows: a) no correlation among the prices of electricity, fuel and $\left.\mathrm{CO}_{2} ; 2\right)$ there are correlations among the prices of electricity, fuel and $\mathrm{CO}_{2}$, and 3) investors can secure a long-term power purchase agreement. We replicate the results of the second scenario. Our results contradict the following conclusion of Roques et al. (2008): "the optimal portfolio for an investor in this case study is to invest only in CCGTs, as any other portfolio would both reduce returns and increase risk."We find efficient portfolios that assign to CCGT a share less than $100 \%$. Actually, the efficient portfolio of minimal risk and also minimal Expected Net Present Value (ENPV) has the following shares: CCGT 53,7\%, nuclear 20,5\%, and coal 25,8\%.

\footnotetext{
${ }^{2}$ Depending on the context, the assets could be technoologies, reactors, or wind production of countries.
} 
Jain et al. (2014) analyze portfolios of four types of nuclear reactors: 1) Generic Gen III type Light Water Reactor (LWR); 2) Fast Reactors (FR); 3) High Temperature Reactor (HTR), and 4) Super Critical Water Reactor (SCWR). The analysis was conducted under budget constraint and capacity constraint. ${ }^{3}$ We replicate the results of the case with budget constraint. The EF we obtain differs considerably from the one obtained by Jain et al. (2014). We find that the portfolio of minimal risk and lower expected return has the following shares: Gen III 18\%, HTR $26 \%$, SCWR 25\%, and FR $31 \%$. This result contradicts the following conclusion of Jain et al. (2014): . ${ }^{\text {nn }}$ investor who wants to minimize the uncertainty of returns and is willing to take a lower expected return in order to do so,will choose a portfolio with more Gen IV type reactors [FR $56 \%$ and HTR $35 \%$ ]."

Roques et al. (2010) analyze cross-country portfolios of joint wind production of five European countries (Austria, Denmark, France, Germany, and Spain). The authors utilize two objective functions to define optimal cross-countries wind power portfolios: i) optimizing wind-power output, and ii) maximizing windpower contribution to system reliability. ${ }^{4}$ We replicate the results of the first objective function. Our results are considerably different than the ones obtained by Roques et al. (2010). For instance, we find that the efficient portfolio that reaches a Wind Portfolio Capacity Factor (WPCF) of 0,2318 allocates the following weights to the five countries: Spain $30 \%$, Germany $8 \%$, Austria 5\%, France 7\%, and Denmark $50 \%$. On the other hand, Roques et al. (2010) find that, for the same WPCF level, the efficient portfolio allocates the following weights to the five countries: Spain 54\%, Germany 0\%, Austria 6\%, France $10 \%$, and Denmark $31 \%$. Differences are noteworthy in the weights received by Denmark, Spain, and Germany.

The present methodology has remarkable advantages: 1) it allows to conduct analysis with up to five assets together; 2) it allows to know the shares of the assets for any risk-return profile; 3 ) the EF is limited by a maximum allowed risk of the PGP; 4) it provides more coherent results than those obtained in the existing literature; 5) it points out that there are optimal investment alternatives that have been denied by previous analysis, and 6) it avoids biases in the design of investment policies in electricity generation. Therefore, the parametric formulation of the EF of the PGP constitutes a powerful tool for both power generation policymakers and large power companies (in liberalized markets). In addition, the present methodology could be extended to analyze portfolios of more than five asses.

The paper also states that the so-called "portfolio effectresults from the fact that the risk of the PGP is a convex function of the shares of the different assets.

The whole analysis relies on the assumption that the covariances of the returns of the different assets are zero. This strong assumption allows gains in tractability, clarity, and in the scope of the methodology formulated.

To the best of our knowledge, this is the first effort to provide a detailed methodology for the parametric construction of the EF of PGP. It is noteworthy that the present parametric formulation of the EF of PGP could be applied to portfolios based on assets different from those of power-generation technologies. ${ }^{5}$

The paper is organized as follows: in Section 2, we present the preliminaries; Section 3 presents PGP of 2 and 3 technologies, replicates the results obtained by Roques et al. (2008), and provides the proof of the "portfolio effect."Section 4 presents PGP of 4 nuclear reactors and replicates the results obtained by Jain et al. (2014). Section 5 presents cross-country PGP of 5 countries and replicates the results obtained by Roques et al. (2010). Section 6 contains the final remarks and conclusions. The appendix contains the formal proofs.

\footnotetext{
${ }^{3}$ See Jain et al. (2014) for the details of the different reactors and the different constraints.

${ }^{4}$ See Roques et al. (2010) for the details of the different objective functions.

${ }^{5}$ For instance, it could be employed to analize portfolios of five electricity suppliers as those studied by Perea González and Zavaleta Vazquez (2020).
} 


\section{Preliminaries}

We apply the Portfolio Theory developed by Markowitz (1952) to find the efficient power-generation mix: the return of the generation mix can be increased only by increasing its risk. As customary, risk is measured by the Standard Deviation (SD) of the returns. Formally, let $X_{i}$ be the random variable that represents the return of asset $i$ and let $Y$ be a random variable describing the return of the PGP, which is defined as follows:

$$
Y=\sum_{i=1}^{n} \alpha_{i} X_{i}, \quad \text { with } \quad \sum_{i=1}^{n} \alpha_{i}=1
$$

Where $\alpha_{i} \in[0,1]$ represents the share of asset $i$. Following result provides the basic tools for the analysis.

Lemma 1 Let $X_{i}$ be a random variable that represents the return of asset $i$ with mean $\mu_{i}$ and variance $\sigma_{i}^{2}$. Where $\alpha_{i} \in[0,1]$ is the the share of asset $i=1,2, \ldots, n$. Let the return of the PGP be represented by the random variable $Y=\sum_{i=1}^{n} \alpha_{i} X_{i}$ with $\sum_{i=1}^{n} \alpha_{i}=1$. Then

$$
\begin{gathered}
E(Y)=\sum_{i=1}^{n} \alpha_{i} E\left(X_{i}\right) \\
\mu_{Y}=\sum_{i=1}^{n} \alpha_{i} \mu_{i} .
\end{gathered}
$$

and

$$
\begin{gathered}
S D(Y)=\sqrt{E\left[Y-\mu_{Y}\right]^{2}}, \\
\sigma_{Y}=\sqrt{\sum_{i=1}^{n} \alpha_{i}^{2} \sigma_{i}^{2}+\sum \sum_{i<j} \alpha_{i} \alpha_{j} \sigma_{i, j}} .
\end{gathered}
$$

where the double summation extends to any values $i$ and $j$, from 1 to $n$, such that $i<j$. In addition, $\sigma_{i, j}=$ $E\left[\left(X_{i}-\mu_{i}\right)\left(X_{j}-\mu_{j}\right)\right]$ is the covariance of the returns of assets $i$ and $j$.

See pp. 158, Freund et al. (2000).

The first result of Lemma 1 indicates that the expected return of the PGP is a convex sum of the expected returns of the different assets. Following corollary summarizes such a fact:

Corollary 1 Assume that assets are ordered according to their expected return, $\mu_{1} \geq \mu_{2} \geq \cdots \geq \mu_{n-1} \geq$ $\mu_{n}$. Then, it holds that $\mu_{1} \geq \mu_{Y} \geq \mu_{n}$.

Second result of Lemma 1 indicates that the risk of the PGP depends on two facts of the different assets, their shares and the covariances of their returns. The risk of the PGP reduces when the covariances among the returns of the assets are negative. The risk of the PGP increases when the covariances among the returns of the assets are positive. ${ }^{6}$ The existing literature reports that, regardless of the variable used to construct the PGP (NPV, LCOE, capacity factor, or installed capacity), the covariances amongst the different assets have an absolute value considerably less than one (e.g., Perea González and Zavaleta Vazquez (2020), Pinheiro Neto et al. (2017), Adams and Jamasb (2016), Cunha and Ferreira (2014), Roques et al. (2010), and Roques et al. (2008)). As the shares of the assets have values less than one, we expect the term $\sum \sum_{i<j} \alpha_{i} \alpha_{j} \sigma_{i, j}$ to be significantly smaller than the term $\sum_{i=1}^{n} \alpha_{i}^{2} \sigma_{i}^{2}$ in the risk of the PGP. This fact is particularly true in two cases: a) when the returns of the PGP are given by the NPVs of the different assets, and b) PGP are based on LCOE of the different assets. Both NPV and LCOE are given in million of dollars or pounds. Therefore, for the present analysis, we assume that the covariances among the different assets is zero, that is, $\sigma_{i, j}=0$, for any values $i$ and $j$, from 1 to $n$, such that $i<j$. Certainly, this assumption leads to a loss in precision in calculating the minimal risk of the PGP. However, such loss is compensated by gains in tractability, clarity, and in the scope of the methodology formulated.

Lemma 1 and Corollary 1 provide the tools to construct the EF of the PGP parametrically. The methodology works as follows:

\footnotetext{
${ }^{6}$ Roques (2006) shows efficient frontier of PGP of CCGT- nuclear for various degrees of hypotetical returns correlation.
} 
1) Rank the assets according to their expected return, $\mu_{1} \geq \mu_{2} \geq \cdots \geq \mu_{n-1} \geq \mu_{n}$. Then, the maximal expected return of the PGP is reached when $\alpha_{1}=1$ and $\alpha_{i}=0$ for $i=2, \ldots, n$.

2) Obtain the shares of the assets, $\alpha_{i}$, that guarantee the minimal risk of the PGP. To do this, we solve the following problem:

$$
\begin{gathered}
\min _{\alpha_{i}} \sigma_{Y}=\sqrt{\sum_{i=1}^{n} \alpha_{i}^{2} \sigma_{i}^{2}} \\
\text { subject to: } \sum_{i=1}^{n} \alpha_{i}=1, \alpha_{i} \geq 0 .
\end{gathered}
$$

3) Formulate the risk of the efficient generation portfolios as a parametric equation of the shares of the assets.

4) Finally, the EF corresponds to the parametric equation of the risk-return profiles from the minimal risk of the PGP to the maximal expected return of the PGP. ${ }^{7}$

Worth noting that, in many cases the EF is not allowed to reach the maximal expected return of the PGP as it is very risky. Under this scenario, we also need to define a maximal allowed risk of the PGP, $\alpha_{1}<1$, and obtain the corresponding shares of the assets and expected return. In this case the EF corresponds to the parametric equation of the risk-return profiles from the minimal risk of the PGP to the maximal allowed risk of the PGP

Now we have all the building blocks to provide a parametric formulation of the EF of PGP. We start with the portfolios of two technologies.

\section{Optimal portfolios for electricity generators in the UK electricity market}

In this section we replicate the results obtained by Roques et al. (2008). The authors apply portfolio theory to identify the portfolios that maximize the returns of private investors for given risk levels in the UK electricity market. The authors use Monte Carlo simulation to compute the distribution of returns and their correlations. Three technologies are considered: CyCle gas turbine (CCGT), coal, and the nuclear plant. Instead of performing the analysis with the three technologies, these authors work with pairs of plants under three scenarios: 1) no correlation among electricity, fuel and $\mathrm{CO}_{2}$ prices; 2) there are correlations between electricity, fuel and $\mathrm{CO}_{2}$ prices, and 3) investors can secure a long-term power purchase agreement.

We replicate the results of the second scenario in which there are correlations among electricity, fuel, and $\mathrm{CO}_{2}$ prices. Due to expositional motives, we conduct the analysis in two steps. First, as Roques et al. (2008) do, we find the efficient portfolios of two technologies: CCGT and coal. Second, we analyze portfolios including those of the three technologies: CCGT, coal and nuclear.

Data is taken from Roques et al. (2008), Table 4, 2nd scenario (with correlations), page 1839. The returns and risk of the different generation technologies are given respectively by the ENPV and the SD of the ENPV. The corresponding ENPV and variance of CCGT (cc), Nuclear (nu) and Coal (co) are: $\mu_{c c}=139, \mu_{n u}=-43$, $\mu_{c o}=-73, \sigma_{c c}^{2}=(233)^{2}=54289, \sigma_{n u}^{2}=(377)^{2}=142129$, and $\sigma_{c o}^{2}=(336)^{2}=112900$.

We proceed to analyze PGP of two technologies.

\footnotetext{
${ }^{7}$ When the PGP is based on LCOE, the ranking of the expected LCOE is as follows: $\mu_{1} \leq \mu_{2} \leq \cdots \leq \mu_{n-1} \leq \mu_{n}$. In this case, the EF corresponds to the parametric equation of the risk-cost profiles from the minimal risk of the PGP to the minimal expected LCOE of the PGP.
} 


\subsection{Portfolios of two technologies}

The risk of the PGP depends on the shares of technologies 1 and 2. The following result provides the shares of technologies 1 and 2 that guarantee the minimal risk of the PGP.

Proposition 1 (Minimal risk of PGP of two technologies) From expression (4), the risk of a PGP of two technologies is given by $\sigma_{Y}=\sqrt{\sum_{i=1}^{2} \alpha_{i}^{2} \sigma_{i}^{2}}$. Assume that the expected return of technology 1 is the least risky. For $\sigma_{1,2}=0, \alpha_{i} \in[0,1]$ for $i=1,2$, and $\alpha_{1}+\alpha_{2}=1$, it holds that

a) The risk of the $P G P$, given by $\sigma_{Y}$, reaches its global minimum at

$$
\left(\begin{array}{c}
\alpha_{1}^{*} \\
\alpha_{2}^{*}
\end{array}\right)=\frac{1}{\sigma_{1}^{2}+\sigma_{2}^{2}}\left(\begin{array}{c}
\sigma_{2}^{2} \\
\sigma_{1}^{2}
\end{array}\right)
$$

b) The minimal risk of the $P G P$ is

$$
\sigma_{Y}^{*}=\sqrt{\left(\sigma_{1}^{2} \sigma_{2}^{2}\right) /\left(\sigma_{1}^{2}+\sigma_{2}^{2}\right)}<\sigma_{1}
$$

See appendix.

The following result provides the parametric formulation of the EF of PGP of two technologies.

Proposition 2 (EF of PGP of two technologies) Let $\sigma_{Y}=\sqrt{\sum_{i=1}^{2} \alpha_{i}^{2} \sigma_{i}^{2}}$ be the risk of the PGP. Assume that $\mu_{1} \geq \mu_{2}$, thus, the following holds:

a) The risk of the efficient portfolios is obtained by the shares of technologies 1 and 2, given by the following parametric equation:

$$
\left(\begin{array}{l}
\alpha_{1} \\
\alpha_{2}
\end{array}\right)=\left(\begin{array}{c}
\alpha \\
1-\alpha
\end{array}\right),
$$

where parameter $\alpha$ is such that $\alpha_{1}^{*} \leq \alpha \leq \alpha^{d f} \leq 1 . \alpha_{1}^{*}$ guarantees the minimal risk of the PGP. While $\alpha^{d f}$ guarantees the maximal allowed risk of the PGP.

b) The risk of the PGP in the EF is given by $\sigma_{Y}(\alpha)=\sqrt{\alpha^{2} \sigma_{1}^{2}+(1-\alpha)^{2} \sigma_{2}^{2}}$, for $\alpha_{1}^{*} \leq \alpha \leq \alpha^{d f}$. Note that $\sigma\left(\alpha^{d f}\right) \leq \sigma_{1}$

c) The maximal expected return for each corresponding level of risk is given by $\mu_{Y}(\alpha)=\alpha \mu_{1}+(1-\alpha) \mu_{2}$, for $\alpha_{1}^{*} \leq \alpha \leq \alpha^{d f}$. Note that $\mu\left(\alpha^{d f}\right) \leq \mu_{1}$.

See appendix.

We now have the theoretical tools to analyze the PGP of CCGT and Coal.

\subsubsection{Efficient portfolios of CCGT-Coal}

Following Proposition 2, CCGT corresponds to technology 1 and Coal to technology 2. Then, from Proposition 1, the shares of the technologies that ensure the minimal risk of the PGP are

$$
\left(\alpha_{c c}^{*}, \alpha_{c o}^{*}\right)=(0,67528,0,32472)
$$

The minimal risk reached by this PGP is $\sigma_{Y}^{*}=191.47$. And the maximal ENPV for such risk is $\mu_{Y}=70,159$. Note that Roques et al. (2008) do not find the minimal risk of the PGP, the shares of the technologies that 
guarantee such a risk, neither these authors find the corresponding ENPV. They only plot the EF of the PGP. ${ }^{8}$

From Proposition 2, the risk of the efficient portfolios is obtained by the shares of technologies 1 and 2 , given by the following parametric equation:

$$
\left(\begin{array}{c}
\alpha_{c c} \\
\alpha_{c o}
\end{array}\right)=\left(\begin{array}{c}
\alpha \\
1-\alpha
\end{array}\right),
$$

for $0,67528 \leq \alpha \leq 1$. Note that the PGP of CCGT-Coal reaches the maximal ENPV when $\alpha=\alpha_{c c}=1$, and $\mu_{Y}=\mu_{c c}=139$. We might say that CCGT "strongly dominates"the PGP, as it has the greatest ENPV, which is also the less risky ENPV. Thus, the risk of the PGP in the efficient frontier is given by 191,47 $\leq$ $\sigma_{y} \leq 233$. The maximal ENPV for each corresponding level of risk is given by $70,159 \leq \mu_{y} \leq 139$. The efficient PGP of CCGT-Coal are shown in the following figure:

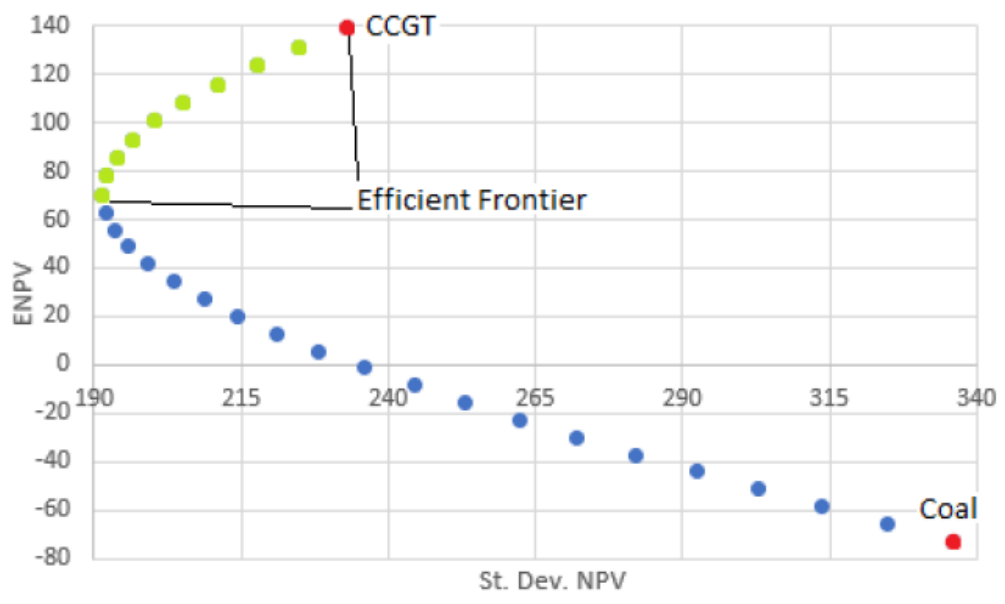

Figure 1. Efficient PGP of CCGT-Coal

The parameters of the EF are presented in the following figure:

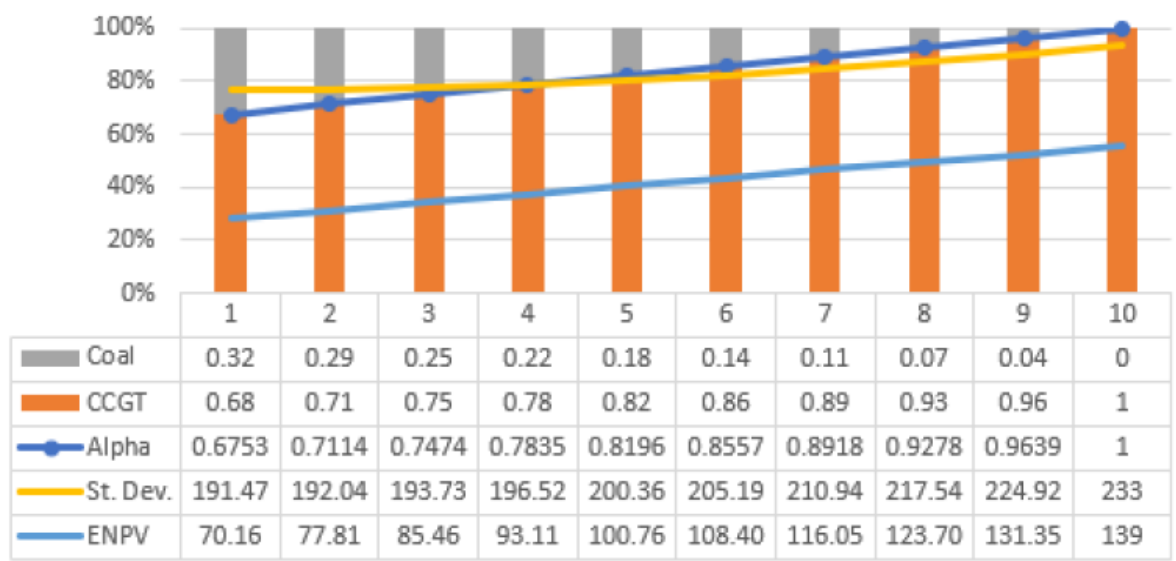

Figure 2. EF of PGP of CCGT-Coal

Although CCGT strongly dominates the PGP, there are efficient portfolios that assign to CCGT a share less than $100 \%$, which reduce return but also reduce risk. Therefore, CCGT receives a share of $68 \%$ in the

\footnotetext{
${ }^{8}$ See Figure 2, page 841, in Roques et al. (2008).
} 
efficient portfolio of minimal risk and also minimal ENPV. This result contradicts the following conclusion of Roques et al. (2008): "The optimal portfolio for an investor in this case study is to invest only in CCGTs, as any other portfolio would both reduce returns and increase risk.Ïn fact, even devoting a share of $100 \%$ to CCGT constitutes an efficient portfolio, an economy would face a potential social risk by placing its power generation in one single technology. Thus a decision-maker might support diversification by, for example, allowing the PGP to reach a risk of $\sigma_{y}=217,54$ and corresponding ENPV $\mu_{y}=123,7$. In such a case, the upper limit of the EF would be given by $\alpha^{d f}=0,92784$ (Figure 2, number 8).

We straightforwardly extend the analysis to the remaining pairs of technologies: CCGT-Nuclear and Nuclear-coal. Following subsection present the EF of every pair of technologies. The corresponding analysis will be provided upon request.

It is noteworthy that the present analysis is incomplete, in that it does not include nuclear plants. The following subsection reveals that introducing nuclear plants changes the shares of the technologies in the efficient portfolios. We now proceed to analyze PGP of three technologies.

\subsection{Portfolios of three technologies}

The following result provides the shares of technologies 1,2 , and 3 , which guarantee the minimum risk of the PGP.

Proposition 3 (Minimal risk of PGP of three technologies) From expression (4), the risk of the PGP of three technologies is given by $\sigma_{Y}=\sqrt{\sum_{i=1}^{3} \alpha_{i}^{2} \sigma_{i}^{2}}$. Assume that the expected return of technology 1 is the least risky. For $\sigma_{1,2}=\sigma_{1,3}=\sigma_{2,3}=0, \alpha_{i} \in[0,1]$ for $i=1,2,3$, and $\sum_{i=1}^{3} \alpha_{i}=1$ it holds that

a) The risk of the $P G P$, given by $\sigma_{Y}$, reaches its global minimum at

$$
\left(\begin{array}{c}
\alpha_{1}^{*} \\
\alpha_{2}^{*} \\
\alpha_{3}^{*}
\end{array}\right)=\frac{1}{\left|A_{3}\right|}\left(\begin{array}{c}
\sigma_{2}^{2} \sigma_{3}^{2} \\
\sigma_{1}^{2} \sigma_{3}^{2} \\
\sigma_{1}^{2} \sigma_{2}^{2}
\end{array}\right),
$$

where $\left|A_{3}\right|=\sigma_{1}^{2} \sigma_{2}^{2}+\sigma_{1}^{2} \sigma_{3}^{2}+\sigma_{2}^{2} \sigma_{3}^{2}$.

b) The minimal risk of the PGP is

$$
\sigma_{Y}^{*}=\sqrt{\left(\sigma_{1}^{2} \sigma_{2}^{2} \sigma_{3}^{2}\right) /\left|A_{3}\right|}<\sigma_{1}
$$

See appendix.

The following result provides the parametric formulation of the EF for PGP of three technologies.

Proposition 4 (EF of PGP of three technologies) Let $\sigma_{Y}=\sqrt{\sum_{i=1}^{3} \alpha_{i}^{2} \sigma_{i}^{2}}$ be the risk of the PGP. Assume that $\mu_{1} \geq \mu_{2} \geq \mu_{3}$, thus, the following holds:

a) The risk of the efficient portfolios is obtained by the shares of technologies 1, 2, and 3, given by the following parametric equation:

$$
\left(\begin{array}{l}
\alpha_{1} \\
\alpha_{2} \\
\alpha_{3}
\end{array}\right)=\left(\begin{array}{c}
\alpha^{1+x} \\
\alpha-\alpha^{1+x} \\
1-\alpha
\end{array}\right),
$$

where parameter $\alpha$ is such that $\left[\alpha_{1}^{*}\right]^{\frac{1}{1+x}} \leq \alpha \leq \alpha^{d f} \leq 1$. Let $x$ be given by $x=\left(\ln \left[\left(\alpha_{1}^{*}\right) /\left(\alpha_{1}^{*}+\alpha_{2}^{*}\right)\right]\right) /\left(\ln \left[\alpha_{1}^{*}+\alpha_{2}^{*}\right]\right)$. $\left[\alpha_{1}^{*}\right]^{\frac{1}{1+x}}$ guarantees the minimal risk of the PGP. While $\alpha^{d f}$ guarantees the maximal allowed risk of the $P G P$. 
b) The risk of the PGP in the EF is given by

$$
\sigma_{Y}(\alpha)=\sqrt{\left[\alpha^{1+x}\right]^{2} \sigma_{1}^{2}+\left[\alpha-\alpha^{1+x}\right]^{2} \sigma_{2}^{2}+[1-\alpha]^{2} \sigma_{3}^{2}}
$$

for $\left[\alpha_{1}^{*}\right]^{\frac{1}{1+x}} \leq \alpha \leq \alpha^{d f}$. Note that $\sigma\left(\alpha^{d f}\right) \leq \sigma_{1}$.

c) The maximal expected return for each corresponding level of risk is given by $\mu_{Y}(\alpha)=\alpha^{1+x} \mu_{1}+\left(\alpha-\alpha^{1+x}\right) \mu_{2}+$ $(1-\alpha) \mu_{3}$, for $\left[\alpha_{1}^{*}\right]^{\frac{1}{1+x}} \leq \alpha \leq \alpha^{d f}$. Note that $\mu\left(\alpha^{d f}\right) \leq \mu_{1}$.

See appendix.

We now have the tools to analyze the PGP of CCGT-Nuclear-Coal.

\subsubsection{Efficient portfolios of CCGT-Nuclear-Coal}

Following Proposition 4, CCGT, nuclear and coal correspond respectively to technologies 1, 2 and 3. Then, from Proposition 3, the shares of the technologies that ensure the minimal risk of the PGP are

$$
\left(\alpha_{c c}^{*}, \alpha_{n u}^{*}, \alpha_{c o}^{*}\right)=(0,53682,0,20505,0,25813)
$$

The minimal risk reached by this PGP is $\sigma_{Y}^{*}=170,71$. The maximal ENPV for such risk is $\mu_{Y}=46,957$. Roques et al. (2008) are not able to find these values because they only carry out the analysis for pairs of technologies.

From Proposition 4, we obtain

$$
x=(\ln [(0,53682) /(0,53682+0,20505)]) /(\ln [0,53682+0,20505])=1.0835
$$

and $\left[\alpha_{1}^{*}\right]^{\frac{1}{1+x}}=[0,53682]^{\frac{1}{2.0835}}=0,74187$. Thus, the risk of the efficient portfolios is obtained by the shares of technologies 1,2 , and 3 , given by the following parametric equation:

$$
\left(\begin{array}{c}
\alpha_{c c} \\
\alpha_{n u} \\
\alpha_{c o}
\end{array}\right)=\left(\begin{array}{c}
\alpha^{2.0835} \\
\alpha-\alpha^{2.0835} \\
1-\alpha
\end{array}\right),
$$

for $0,74187 \leq \alpha \leq 1$. The PGP of CCGT-Coal-Nuclear reaches the maximal ENPV when $\alpha=\alpha_{c c}=1$, and $\mu_{Y}=\mu_{c c}=139$. Again, we might say that CCGT "strongly dominates"the PGP, as it has the greatest ENPV and the lowest risk. The risk of the PGP in the EF is given by $170,71 \leq \sigma_{y} \leq 233$. The maximal ENPV for each corresponding risk is given by $46,957 \leq \mu_{y} \leq 139$. The efficient PGP of Nuclear-Coal-CCGT are depicted in the following figure: 


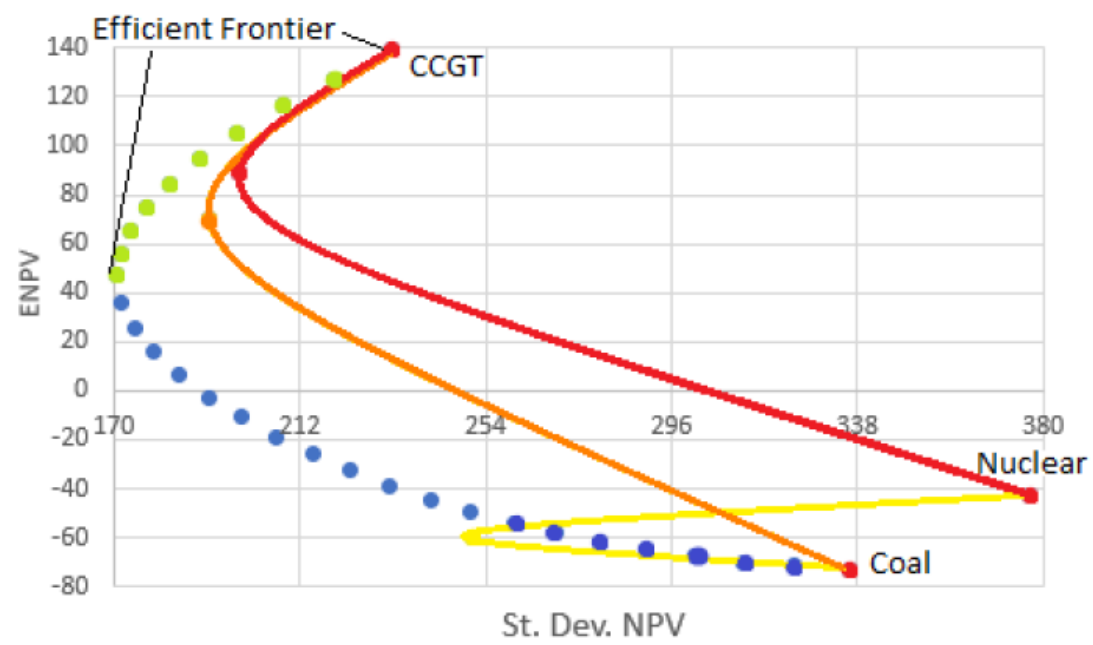

Figure 3. Efficient PGP of Nuclear-Coal-CCGT

The triangle in Figure 3 resembles the triangle of the left side of Figure 2, page 841, in Roques et al. (2008). However, alike the triangle in Roques et al. (2008), we include the PGP of minimal risk for each pair of technologies. The parameters of the EF of the PGP are presented in figure 4.

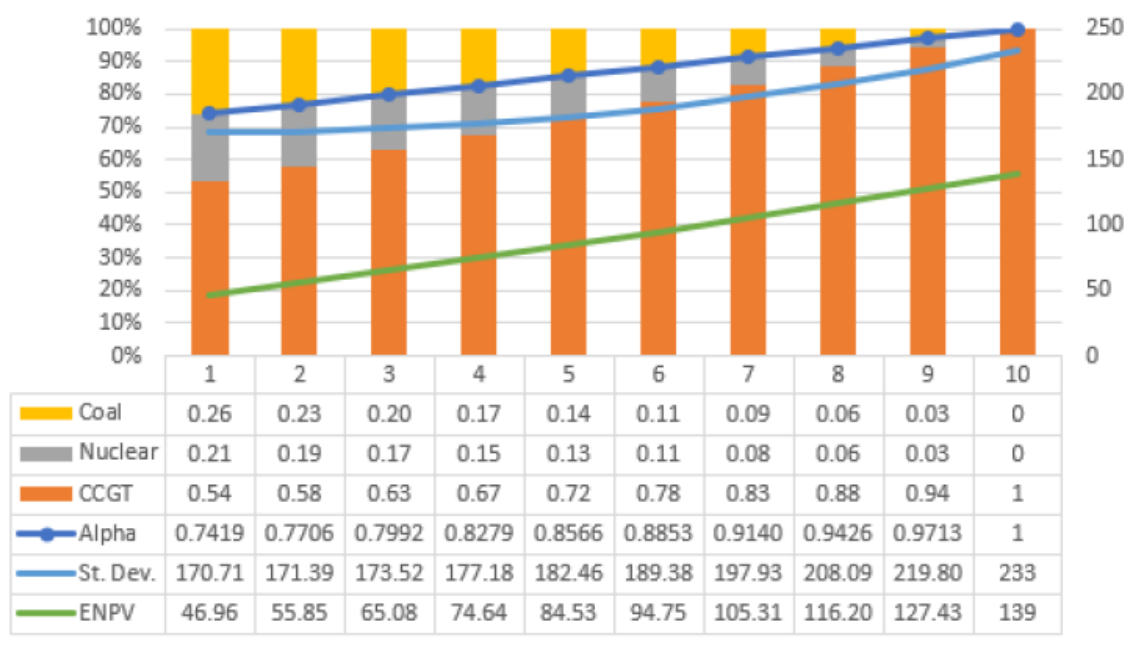

Figure 4. EF of PGP of Nuclear-Coal-CCGT

We find that, although CCGT strongly dominates the PGP, there are efficient portfolios that assign to CCGT a share less than $100 \%$, that reduce return but also reduce risk. In fact, the efficient portfolio of minimal risk and also minimal ENPV has the following shares: CCGT 53,7\%, nuclear 20,5\%, and coal $25,8 \%$. This result contradicts the following conclusion of Roques et al. (2008): "The optimal portfolio for an investor in this case study is to invest only in CCGTs, as any other portfolio would both reduce returns and increase risk.. ${ }^{\mathrm{A}}$ gain, a decision-maker might support diversification by, for example, allowing the PGP to reach a risk of $\sigma_{y}=208,09$ and corresponding ENPV $\mu_{y}=116,2$. In such a case, the upper limit of the efficient frontier would be given by $\alpha^{d f}=0,94263$ (Figure 4, number 8). We find our results more coherent than those obtained by Roques et al. (2008).

The present methodology permits the analysis of the PGP of three technologies, providing the shares of the technologies for any risk-return profile in the EF. Thus, there is no need to apply any mean-variance utility function, as Roques et al. (2008) do, in order to find efficient portfolios for certain levels of risk aversion. 
Actually, it would be straightforward to verify that the mean-variance utility function used by Roques et al. (2008) reaches its maximum at the efficient portfolio of minimal risk and also minimal ENPV $\left(\sigma_{Y}^{*}=170,71\right.$ and $\left.\mu_{Y}=46,957\right)$.

\subsection{The portfolio effect}

It is worth noting that the minimal risk of the PGP of CCGT-coal is $\sigma_{Y_{2}}^{*}=191.47$, while the minimal risk of the PGP of CCGT-Coal-Nuclear is $\sigma_{Y_{3}}^{*}=170.71$. This is, the minimal risk of a portfolio of three assets is lower than the minimal risk of a portfolio of only two assets. This fact is not a coincidence, it is due to the so called "portfolio effect": risk reduction due to diversification (Awerbuch and Berger (2003)). The following result states that the portfolio effect arises from the fact that the risk of the PGP is a convex function of the shares of the different technologies.

Proposition 5 (Portfolio effect) Let $\sigma_{Y}=\sqrt{\sum_{i=1}^{n} \alpha_{i}^{2} \sigma_{i}^{2}}$ be the risk of the PGP of $n$ technologies. Let $\sigma_{Y_{n}}^{*}$ be the minimal risk reached by the portfolio of $n$ technologies. Let $\sigma_{Y_{k}}^{*}$ be the minimal risk reached by a portfolio of $k$ of the $n$ available technologies, $k<n$. Then, the following holds:

$$
\sigma_{Y_{n}}^{*}<\sigma_{Y_{k}}^{*}
$$

See appendix.

The previous result states that there is no need to include a risk-free asset to reduce the risk of a PGP, as argued by Awerbuch and Berger (2003).

Thus, from Proposition 5, we conclude that the results obtained by Roques et al. (2008) are incomplete, in that they were derived by analyzing only pairs of the three available technologies. Therefore, their conclusions create a bias in the design of investment policies in electricity generation. ${ }^{9}$

\section{Nuclear reactor generation portfolios}

In this section we replicate the results obtained by Jain et al. (2014). The authors apply analyze portfolios of nuclear reactors that maximize the returns for given risk levels. They use real options to compute the distribution of the returns. The following four types of nuclear reactors were considered for the portfolio: 1) Generic Gen III type Light Water Reactor (LWR); 2) Fast Reactors (FR); 3) High Temperature Reactor (HTR), and 4) Super Critical Water Reactor (SCWR). The analysis was conducted under budget constraint and capacity constraint. In this section, we replicate the results of the case with budget constraint.

The risk of the portfolio depends on the shares of reactors 1,2,3 and 4 . The following result provides the shares of reactors $1,2,3$, and 4 that guarantee the minimal risk of the PGP.

Proposition 6 (Minimal risk of PGP of four reactors) From expression (4), the risk of the PGP of four reactors is given by $\sigma_{Y}=\sqrt{\sum_{i=1}^{4} \alpha_{i}^{2} \sigma_{i}^{2}}$. Assume that the expected return of reactor 4 is the least risky. Assume that $\sigma_{i, j}=0$, for any values $i$ and $j$, from 1 to 4 , such that $i<j$. If $\alpha_{i} \in[0,1]$ for $i=1,2,3,4$, and $\sum_{i=1}^{4} \alpha_{i}=1$ it holds that

\footnotetext{
${ }^{9}$ In this regard, Gómez-Ríos and Juárez-Luna (2019) also analyze pairs of three available technologies. Then, their results are also incomplete.
} 
a) The risk of the $P G P, \sigma_{Y}$, reaches its global minimum at

$$
\left(\begin{array}{c}
\alpha_{1}^{*} \\
\alpha_{2}^{*} \\
\alpha_{3}^{*} \\
\alpha_{4}^{*}
\end{array}\right)=\frac{1}{\left|A_{4}\right|}\left(\begin{array}{c}
\sigma_{2}^{2} \sigma_{3}^{2} \sigma_{4}^{2} \\
\sigma_{1}^{2} \sigma_{3}^{2} \sigma_{4}^{2} \\
\sigma_{1}^{2} \sigma_{2}^{2} \sigma_{4}^{2} \\
\sigma_{1}^{2} \sigma_{2}^{2} \sigma_{3}^{2}
\end{array}\right) .
$$

where $\left|A_{4}\right|=\sigma_{1}^{2} \sigma_{2}^{2} \sigma_{3}^{2}+\sigma_{1}^{2} \sigma_{2}^{2} \sigma_{4}^{2}+\sigma_{1}^{2} \sigma_{3}^{2} \sigma_{4}^{2}+\sigma_{2}^{2} \sigma_{3}^{2} \sigma_{4}^{2}$.

b) The minimal risk of the $P G P$ is

$$
\sigma_{Y}^{*}=\sqrt{\left(\sigma_{1}^{2} \sigma_{2}^{2} \sigma_{3}^{2} \sigma_{4}^{2}\right) /\left|A_{4}\right|}<\sigma_{4}
$$

See appendix.

The following result provides the parametric formulation of the efficient frontier for PGP of four reactors.

Proposition 7 (EF of PGP of four reactors) Let $\sigma_{Y}=\sqrt{\sum_{i=1}^{4} \alpha_{i}^{2} \sigma_{i}^{2}}$ be the risk of the portfolio. Assume that $\mu_{1} \geq \mu_{2} \geq \mu_{3} \geq \mu_{4}$, thus, the following holds:

a) The risk of the efficient portfolios is obtained by the shares of reactors 1,2,3, and 4, given by the following parametric equation:

$$
\left(\begin{array}{c}
\alpha_{1} \\
\alpha_{2} \\
\alpha_{3} \\
\alpha_{4}
\end{array}\right)=\left(\begin{array}{c}
\alpha^{1+x_{1}} \\
\alpha-\alpha^{1+x_{1}} \\
\alpha^{x_{2}}-\alpha^{1+x_{2}} \\
1-\alpha-\alpha^{x_{2}}+\alpha^{1+x_{2}}
\end{array}\right),
$$

where parameter $\alpha$ is such that $\left[\alpha_{1}^{*}\right]^{\frac{1}{1+x_{1}}} \leq \alpha \leq \alpha^{d f} \leq 1$. Let $x_{1}$ and $x_{2}$ be given by

$$
\begin{aligned}
& x_{1}=\left(\ln \left[\left(\alpha_{1}^{*}\right) /\left(\alpha_{1}^{*}+\alpha_{2}^{*}\right)\right]\right) /\left(\ln \left[\alpha_{1}^{*}+\alpha_{2}^{*}\right]\right), \text { and } \\
& x_{2}=\left(\ln \left[\left(\alpha_{3}^{*}\right) /\left(\alpha_{3}^{*}+\alpha_{4}^{*}\right)\right]\right) /\left(\ln \left[\alpha_{1}^{*}+\alpha_{2}^{*}\right]\right) .
\end{aligned}
$$

$\left[\alpha_{1}^{*}\right]^{\frac{1}{1+x_{1}}}$ guarantees the minimal risk of the PGP. While $\alpha^{d f}$ guarantees the maximal allowed risk of the $P G P$.

b) The risk of the PGP in the EF is given by

$$
\sigma_{Y}(\alpha)=\sqrt{\left[\alpha^{x_{1}+1}\right]^{2} \sigma_{1}^{2}+\left[\alpha-\alpha^{x_{1}+1}\right]^{2} \sigma_{2}^{2}+\left[\alpha^{x_{2}}-\alpha^{x_{2}+1}\right]^{2} \sigma_{3}^{2}+\left[1-\alpha-\alpha^{x_{2}}+\alpha^{x_{2}+1}\right]^{2} \sigma_{4}^{2}} .
$$

for $\left[\alpha_{1}^{*}\right]^{\frac{1}{1+x_{1}}} \leq \alpha \leq \alpha^{d f}$. Note that $\sigma\left(\alpha^{d f}\right) \leq \sigma_{1}$.

c) The maximal expected return for every corresponding level of risk is given by

$$
\mu_{Y}(\alpha)=\alpha^{1+x_{1}} \mu_{1}+\left(\alpha-\alpha^{1+x_{1}}\right) \mu_{2}+\left(\alpha^{x_{2}}-\alpha^{1+x_{2}}\right) \mu_{3}+\left(1-\alpha-\alpha^{x_{2}}+\alpha^{1+x_{2}}\right) \mu_{4}
$$

for $\left[\alpha_{1}^{*}\right]^{\frac{1}{1+x_{1}}} \leq \alpha \leq \alpha^{d f}$. Note that $\mu\left(\alpha^{d f}\right) \leq \mu_{1}$.

See appendix.

We already have the theoretical tools to replicate the results obtained by Jain et al. (2014) for the nuclear portfolio of Gen III-HTR-SCWR-FR, under budget constraint. Data is taken from Jain et al. (2014), Table 11, page 108. The expected returns and variance of reactors Gen III, HTR, SCWR and FR are, 
respectively: $\mu_{\text {GenIII }}=1,3259, \mu_{H T R}=1,0959, \mu_{S C W R}=0,972, \mu_{F R}=0,30778, \sigma_{\text {GenIII }}^{2}=(1,3036)^{2}=1$. $6994, \sigma_{H T R}^{2}=(1,0873)^{2}=1,1822, \sigma_{S C W R}^{2}=(1,1184)^{2}=1,2508$, and $\sigma_{F R}^{2}=(0,99925)^{2}=0,9985$.

Following Proposition 7, Gen III, HTR, SCWR, and FR correspond, respectively, to reactors 1, 2, 3, and 4. Thus, from Proposition 6, the shares of the four reactors that ensure the minimal risk are

$$
\left(\alpha_{G e n I I I}^{*}, \alpha_{H T R}^{*}, \alpha_{S C W R}^{*}, \alpha_{F R}^{*}\right)=(0,18188,0,26145,0,24711,0,30955) .
$$

The minimal risk reached by this PGP is $\sigma_{Y}^{*}=0,55596$. The maximal expected return for such a risk is $\mu_{Y}=$ 0,86314 . These values are notably different from those obtained by Jain et al. (2014). These authors find that the minimal risk of the portfolio is 0,95 while the corresponding expected return to such a level of risk is 0,6 . These authors also indicate, in Figure 7, that the portfolio of minimal risk and also the minimal return has the following (estimated) ${ }^{10}$ shares: $\left(\alpha_{\text {GenIII }}, \alpha_{H T R}, \alpha_{S C W R}, \alpha_{F R}\right)=(0,0,08,0,37,0,55)$.

Following Proposition 7, we obtain

$$
\begin{aligned}
& x_{1}=(\ln [(0,18188) /(0,18188+0,26145)]) /(\ln [0,18188+0,26145])=1.0953 \\
& x_{2}=(\ln [(0,24711) /(0,24711+0,30955)]) /(\ln [0,18188+0,26145])=0,99838
\end{aligned}
$$

and $\left[\alpha_{1}^{*}\right]^{\frac{1}{1+x_{1}}}=[0,18188]^{\frac{1}{1+1.0953}}=0,44333$. Thus, the risk of the efficient portfolios is obtained by the shares of reactors $1,2,3$, and 4 , given by the following parametric equation:

$$
\left(\begin{array}{c}
\alpha_{\text {GenIII }} \\
\alpha_{H T R} \\
\alpha_{S C W R} \\
\alpha_{F R}
\end{array}\right)=\left(\begin{array}{c}
\alpha^{2.0953} \\
\alpha-\alpha^{2.0953} \\
\alpha^{0,99838}-\alpha^{1,99838} \\
1-\alpha-\alpha^{0,99838}+\alpha^{1,99838}
\end{array}\right)
$$

for $0,44333 \leq \alpha \leq 0,8739065$. The PGP of Gen III-HTR-SCWR-FR reaches the maximal expected return when $\alpha=\alpha_{\text {GenIII }}=1$, and $\mu_{Y}=\mu_{G e n I I I}=1,3259$. However, to support diversification, the PGP might be allowed to reach a risk of $\sigma_{F R}=0,99925$, which is the minimal risk of the four nuclear reactor. In this case, the corresponding expected return is $\mu_{y}=11,243144281$ and the upper limit of the efficient frontier would be given by $\alpha^{d f}=0,8739065$. Thus, the risk of the PGP in the EF is given by $0,55596 \leq \sigma_{y} \leq 0,99925$. The maximal expected return for every corresponding risk is given by $0,86314 \leq \mu_{y} \leq 1,243144281$. The efficient PGPs of Gen III-HTR-SCWR-FR are presented in Figure 5.

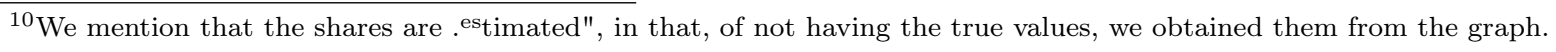




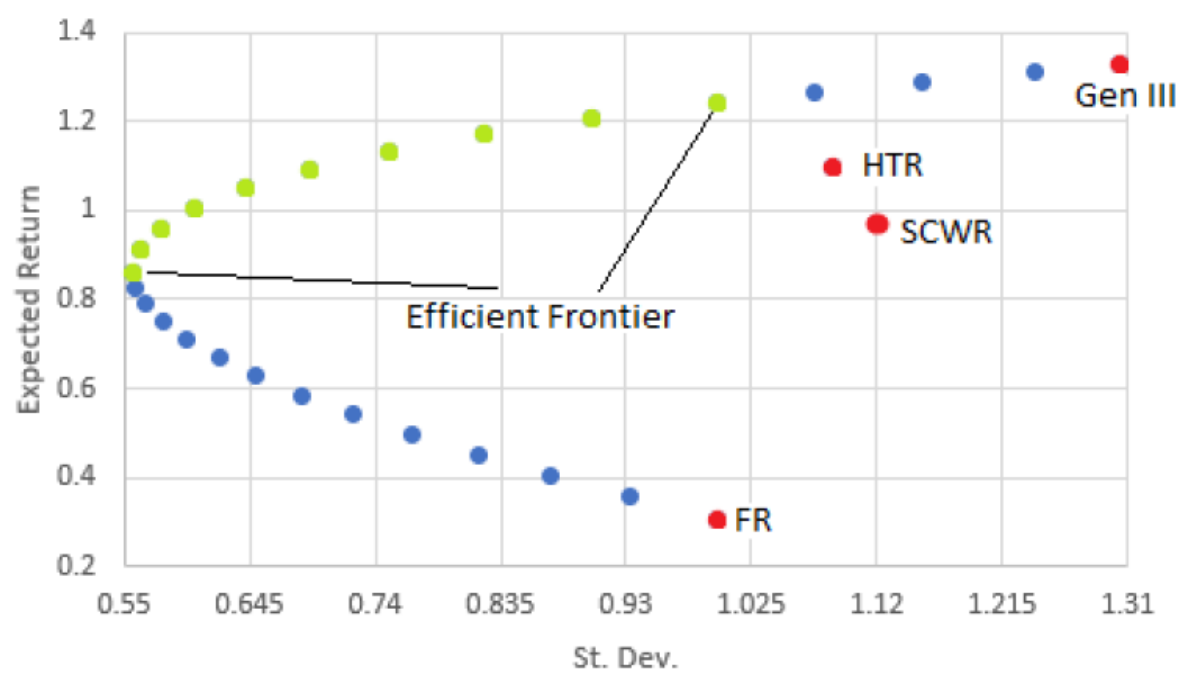

Figure 5. Efficient PGP of Gen III-HTR-SCWR-FR

The following figure presents the parameters of the EF. The proposed EF ranges from 1 to 10. Notwithstanding this, we present all of the efficient portfolios in order to compare them with those obtained by Jain et al. (2014), shown in figure $7 \mathrm{~b}$ (page 108).

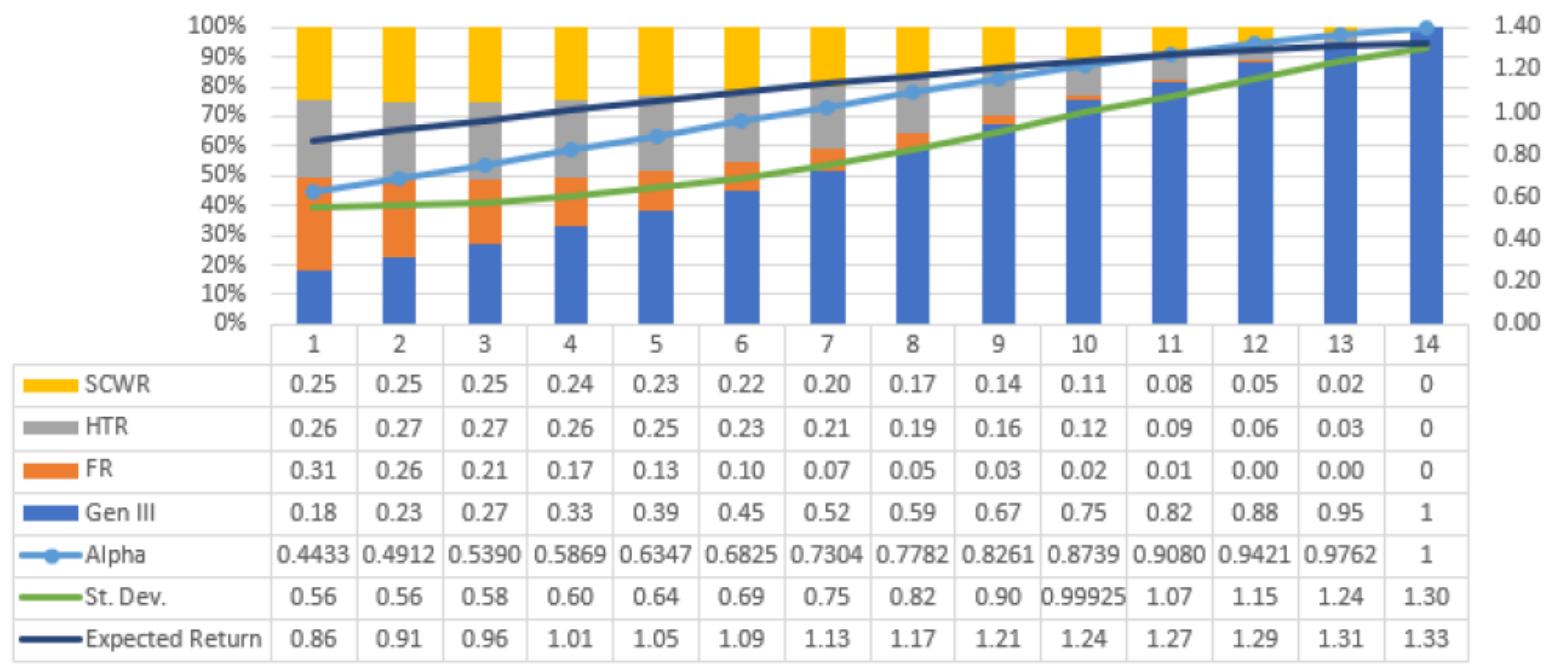

Figure 6. EF of PGP of Gen III-HTR-SCWR-FR

We find that an investor who wants to minimize risk with a lower expected return will chose a portfolio with following shares: Gen III 18\%, HTR 26\%, SCWR 25\%, and FR 31\%. This result contradicts the following conclusion of Jain et al. (2014): . ${ }^{\mathrm{A}} \mathrm{n}$ investor who wants to minimize the uncertainty of returns and is willing to take a lower expected return in order to do so,will choose a portfolio with more Gen IV type reactors [FR $56 \%$ and HTR $35 \%$ ].. ${ }^{\circ}$ ur EF differs considerably from that obtained by Jain et al. (2014). For example, Jain et al. (2014) state, in figure 7, that the efficient portfolio that reaches an expected return of 1,2 , allocates to reactors Gen III and HTR a share of around $50 \%$. The present analysis indicates that, for such an expected return, the shares of the four reactors are: Gen III $67 \%$, HTR 16\%, SCWR 14\%, and FR $3 \%$. We find our results more coherent than those obtained by Jain et al. (2014).

When replicating the case under capacity constraint, we also find different results from those of Jain et al. (2014). For example, Jain et al. (2014) state, in figure 8, that the efficient portfolio of minimum risk 
$\left(\sigma_{Y}^{*}=2800\right)$ and also minimum expected return $\left(\mu_{Y}=2800\right)$ has the following (estimated) shares: Gen III $43 \%$, HTR $8 \%$, SCWR $42 \%$, and FR $7 \%$. On the other hand, we find that the efficient portfolio of minimal risk $\left(\sigma_{Y}^{*}=1584,42\right)$ and also minimum expected return $\left(\mu_{Y}=2601,18\right)$ has the following shares: Gen III $27 \%$, HTR $21 \%$, SCWR $28 \%$, and FR $24 \%$ (This case will be provided upon request).

It is noteworthy that the difference in the results creates a bias in the design of investment policies in electricity generation by nuclear reactors.

Jain et al. (2014) also test the sensitivity of the portfolio under three different scenarios: 1) varying discount rates; 2) varying electricity price growth rates, and 3) varying uncertainties in electricity prices. However, they do not provide the statistics of the returns for such scenarios. The present methodology could be applied to replicate the results of such scenarios if the statistics of the returns were available.

\section{Optimal portfolios of wind-power in Europe}

In this section, we replicate the results obtained by Roques et al. (2010). These authors use historical windproduction data from five European countries (Austria, Denmark, France, Germany, and Spain) to identify cross-country portfolios that minimize the risk of joint wind production for a given level of production. They employ two objective functions to define optimal cross-country wind power portfolios: i) optimizing windpower output, and ii) maximizing wind-power contribution to system reliability. In this section, we replicate the results of the first case.

The risk of the portfolio depends on the weight received by the wind production of countries $1,2,3,4$, and 5 . The following result provides the weights received by countries $1,2,3,4$, and 5 that guarantee the minimal risk of the PGP.

Proposition 8 (Minimal risk of PGP of five countries) From expression (4), the risk of the PGP of five countries is given by $\sigma_{Y}=\sqrt{\sum_{i=1}^{5} \alpha_{i}^{2} \sigma_{i}^{2}}$. Assume that the expected return of country 3 is the least risky. Assume that $\sigma_{i, j}=0$, for any values $i$ and $j$, from 1 to 5 , such that $i<j$. If $\alpha_{i} \in[0,1]$ for $i=1,2,3,4,5$, and $\sum_{i=1}^{5} \alpha_{i}=1$ it holds that

a) The risk of the $P G P, \sigma_{Y}$, reaches its global minimum at

$$
\left(\begin{array}{c}
\alpha_{1}^{*} \\
\alpha_{2}^{*} \\
\alpha_{3}^{*} \\
\alpha_{4}^{*} \\
\alpha_{5}^{*}
\end{array}\right)=\frac{1}{\left|A_{5}\right|}\left(\begin{array}{c}
\sigma_{2}^{2} \sigma_{3}^{2} \sigma_{4}^{2} \sigma_{5}^{2} \\
\sigma_{1}^{2} \sigma_{3}^{2} \sigma_{4}^{2} \sigma_{5}^{2} \\
\sigma_{1}^{2} \sigma_{2}^{2} \sigma_{4}^{2} \sigma_{5}^{2} \\
\sigma_{1}^{2} \sigma_{2}^{2} \sigma_{3}^{2} \sigma_{5}^{2} \\
\sigma_{1}^{2} \sigma_{2}^{2} \sigma_{3}^{2} \sigma_{4}^{2}
\end{array}\right) .
$$

where $\left|A_{5}\right|=\sigma_{1}^{2} \sigma_{2}^{2} \sigma_{3}^{2} \sigma_{4}^{2}+\sigma_{1}^{2} \sigma_{2}^{2} \sigma_{3}^{2} \sigma_{5}^{2}+\sigma_{1}^{2} \sigma_{2}^{2} \sigma_{4}^{2} \sigma_{5}^{2}+\sigma_{1}^{2} \sigma_{3}^{2} \sigma_{4}^{2} \sigma_{5}^{2}+\sigma_{2}^{2} \sigma_{3}^{2} \sigma_{4}^{2} \sigma_{5}^{2}$.

b) The minimal risk of the PGP is

$$
\sigma_{Y}^{*}=\sqrt{\left(\sigma_{1}^{2} \sigma_{2}^{2} \sigma_{3}^{2} \sigma_{4}^{2} \sigma_{5}^{2}\right) /\left|A_{5}\right|}<\sigma_{3}
$$

See appendix.

Following result provides the parametric formulation of the EF of PGP of cross-country wind power.

Proposition 9 (EF of PGP of five countries) Let $\sigma_{Y}=\sqrt{\sum_{i=1}^{5} \alpha_{i}^{2} \sigma_{i}^{2}}$ be the risk of the PGP. Assume that $\mu_{1} \geq \mu_{2} \geq \mu_{3} \geq \mu_{4} \geq \mu_{5}$, thus, the following holds: 
a) The risk of the efficient portfolios is obtained by the weight received by the wind production of countries $1,2,3,4$, and 5 , given by the following parametric equation:

$$
\left(\begin{array}{l}
\alpha_{1} \\
\alpha_{2} \\
\alpha_{3} \\
\alpha_{4} \\
\alpha_{5}
\end{array}\right)=\left(\begin{array}{c}
\alpha^{1+x_{1}+x_{2}} \\
\alpha^{1+x_{2}}-\alpha^{1+x_{1}+x_{2}} \\
\alpha^{1+x_{3}}-\alpha^{1+x_{2}+x_{3}} \\
\alpha-\alpha^{1+x_{2}}-\alpha^{1+x_{3}}+\alpha^{1+x_{2}+x_{3}} \\
1-\alpha
\end{array}\right)
$$

where the parameter $\alpha$ is such that $\left[\alpha_{1}^{*}\right]^{\frac{1}{1+x_{1}+x_{2}}} \leq \alpha \leq \alpha^{d f} \leq 1$.

Let $x_{1}, x_{2}$, and $x_{3}$ be given by

$$
\begin{aligned}
& x_{1}=\left(\ln \left[\left(\alpha_{1}^{*}\right) /\left(\alpha_{1}^{*}+\alpha_{2}^{*}\right)\right]\right) /\left(\ln \left[1-\alpha_{5}^{*}\right]\right), \\
& x_{2}=\left(\ln \left[\left(\alpha_{1}^{*}+\alpha_{2}^{*}\right) /\left(1-\alpha_{5}^{*}\right)\right]\right) /\left(\ln \left[1-\alpha_{5}^{*}\right]\right), \text { and } \\
& x_{3}=\left(\ln \left[\left(\alpha_{3}^{*}\right) /\left(\alpha_{3}^{*}+\alpha_{4}^{*}\right)\right]\right) /\left(\ln \left[1-\alpha_{5}^{*}\right]\right)
\end{aligned}
$$

$\left[\alpha_{1}^{*}\right]^{\frac{1}{1+x_{1}+x_{2}}}$ guarantees the minimal risk of the PGP, while $\alpha^{d f}$ guarantees the maximal allowed risk of the $P G P$.

b) The risk of the PGP in the EF is given by

$$
\begin{aligned}
& \sigma_{Y}(\alpha)=\left\{\left[\alpha^{1+x_{1}+x_{2}}\right]^{2} \sigma_{1}^{2}+\left[\alpha^{1+x_{2}}-\alpha^{1+x_{1}+x_{2}}\right]^{2} \sigma_{2}^{2}+\left[\alpha^{1+x_{3}}-\alpha^{1+x_{2}+x_{3}}\right]^{2} \sigma_{3}^{2}+\left[\alpha-\alpha^{1+x_{2}}-\alpha^{1+x_{3}}+\alpha^{1+x_{2}+x_{3}}\right]^{2} \sigma_{4}^{2}\right. \\
& \text { for }\left[\alpha_{1}^{*}\right]^{\frac{1}{1+x_{1}+x_{2}}} \leq \alpha \leq \alpha^{d f} . \text { Note that } \sigma\left(\alpha^{d f}\right) \leq \sigma_{1}
\end{aligned}
$$

c) The maximal expected return for every corresponding level of risk is given by

$$
\mu_{Y}(\alpha)=\left(\alpha^{1+x_{1}+x_{2}}\right) \mu_{1}+\left(\alpha^{1+x_{2}}-\alpha^{1+x_{1}+x_{2}}\right) \mu_{2}+\left(\alpha^{1+x_{3}}-\alpha^{1+x_{2}+x_{3}}\right) \mu_{3}+\left(\alpha-\alpha^{1+x_{2}}-\alpha^{1+x_{3}}+\alpha^{1+x_{2}+x_{3}}\right) \mu_{4}+(1
$$

for $\left[\alpha_{1}^{*}\right]^{\frac{1}{1+x_{1}+x_{2}}} \leq \alpha \leq \alpha^{d f}$. Note that $\mu\left(\alpha^{d f}\right) \leq \mu_{1}$.

See appendix.

We now possessed the theoretical tools to replicate the results obtained by Roques et al. (2010) for the wind portfolios of Denmark-Austria-Spain-France-Germany under the case optimizing wind power output. Data is taken from Roques et al. (2010), Table 5, page 3250. For these the returns and the risk of the different countries are given by the wind-power capacity factors (WPCF) and their SD. The corresponding expected returns and variance of Denmark, Austria, Spain, France, and Germany are: $\mu_{D n}=0,242, \mu_{A u}=0,229$, $\mu_{S p}=0,229, \mu_{F r}=0,214, \mu_{G r}=0,195, \sigma_{D n}^{2}=(0,027)^{2}=7.29 \times 10^{-4}, \sigma_{A u}^{2}=(0,048)^{2}=2.304 \times 10^{-3}$, $\sigma_{S p}^{2}=(0,016)^{2}=2.56 \times 10^{-4}, \sigma_{F r}^{2}=(0,017)^{2}=2.89 \times 10^{-4}$, and $\sigma_{G r}^{2}=(0,019)^{2}=3.61 \times 10^{-4}$.

From Proposition 9, countries 1, 2, 3, 4, and 5 correspond, respectively, to Denmark, Austria, Spain, France, and Germany. Thus, from Proposition 8, the weights received by the wind production of the countries that ensure the minimal risk are

$$
\left(\alpha_{D n}^{*}, \alpha_{A u}^{*}, \alpha_{S p}^{*}, \alpha_{F r}^{*}, \alpha_{G r}^{*}\right)=(0,11486,0,036343,0,32709,0,28974,0,23195) .
$$

The minimal risk of this PGP is given by $\sigma_{Y}^{*}=0,0091507$. And the maximal expected WPCF for such level of risk is $\mu_{Y}=0,21826$. These values are different from those obtained by Roques et al. (2010). These authors find 
that the minimal risk of the portfolio is somewhat greater that 0,01 while the corresponding expected return to such a level of risk is 0,2190 . They also indicate, in Figure 4, that the portfolio of minimal risk and also the minimal return has the following (estimated) weights: $\left(\alpha_{D n}, \alpha_{A u}, \alpha_{S p}, \alpha_{F r}, \alpha_{G r}\right)=(0,09,0,03,0,39,0,29,0,2)$.

From Proposition 9, we obtain

$$
\begin{aligned}
& x_{1}=(\ln [(0,11486) /(0,11486+0,036343)]) /(\ln [1-0,23195])=1.0417, \\
& x_{2}=(\ln [(0,11486+0,036343) /(1-0,23195)]) /(\ln [1-0,23195])=6.1585, \\
& x_{3}=(\ln [(0,32709) /(0,32709+0,28974)]) /(\ln [1-0,23195])=2.4038,
\end{aligned}
$$

and $\left[\alpha_{1}^{*}\right]^{\frac{1}{1+x_{1}+x_{2}}}=[0,11486]^{\frac{1}{1+1,0417+6.1585}}=0,76805$. Thus, the risk of the efficient portfolios is obtained by the weights received by the wind production of countries $1,2,3,4$ and 5 , given by the following parametric equation:

$$
\left(\begin{array}{c}
\alpha_{D n} \\
\alpha_{A u} \\
\alpha_{S p} \\
\alpha_{F r} \\
\alpha_{G r}
\end{array}\right)=\left(\begin{array}{c}
\alpha^{8.2002} \\
\alpha^{7.1585}-\alpha^{8.2002} \\
\alpha^{3.4038}-\alpha^{9.5623} \\
\alpha-\alpha^{7.1585}-\alpha^{3.4038}+\alpha^{9.5623} \\
1-\alpha
\end{array}\right)
$$

for $0,76805 \leq \alpha \leq 0,94105$. The PGP of Denmark-Austria-Spain-France-Germany reaches the maximal WPCF when $\alpha=\alpha_{D n}=1$, and $\mu_{Y}=\mu_{D n}=0,242$. However, to support diversification, the PGP might be allowed to reach a risk of $\sigma_{F R}=0,17$, that is, one of the less risky countries. In this case, the corresponding WPCF is $\mu_{y}=0,2343$ and the upper limit of the EF would be given by $\alpha^{d f}=0,94105$. Thus, the risk of the PGP in the EF is given by $0,0091507 \leq \sigma_{y} \leq 0,17$. The maximal expected return for each corresponding risk is given by $0,21826 \leq \mu_{y} \leq 0,2343$. The following figure presents the efficient PGP of cross-country wind-power.

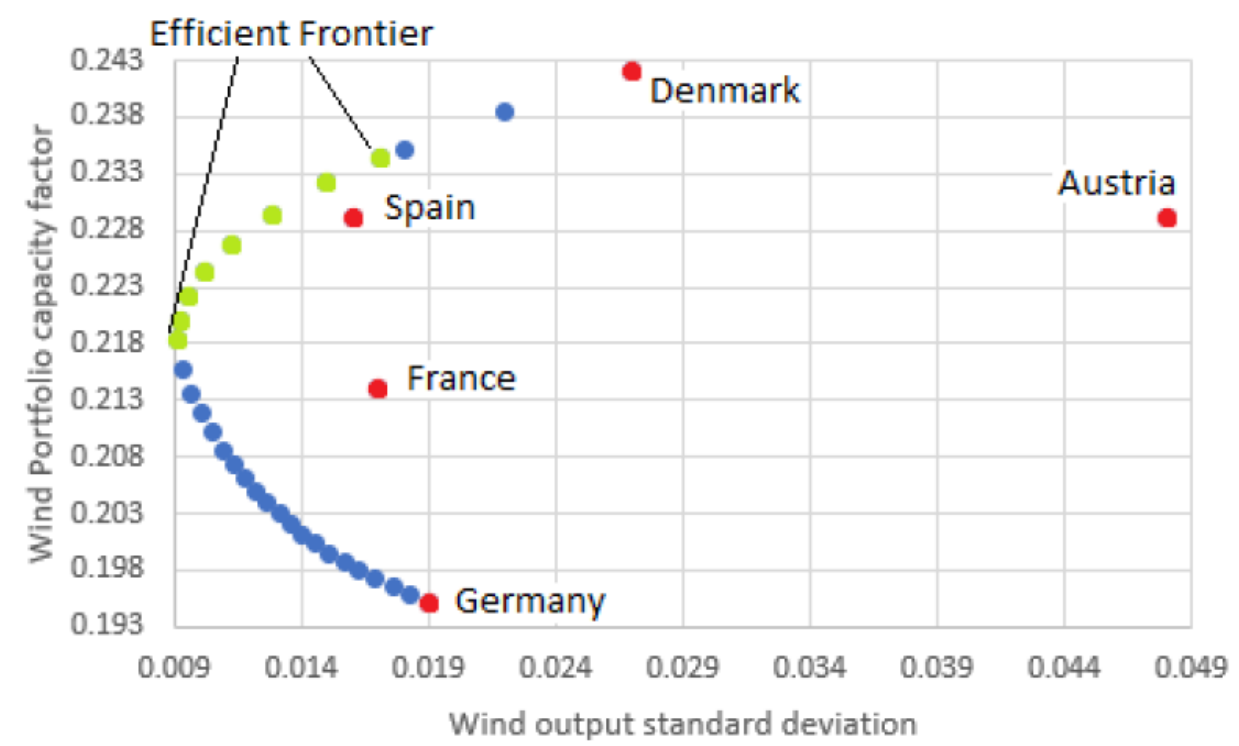

Figure 7. Efficient PGP, Dn-Au-Sp-Fr-Gr

The following figure presents the parameters of the EF. The proposed EF ranges from 1 to 8. However, we present all of the efficient portfolios in order to compare them with those obtained by Roques et al. (2010), shown in figure 4 (page 3251). 


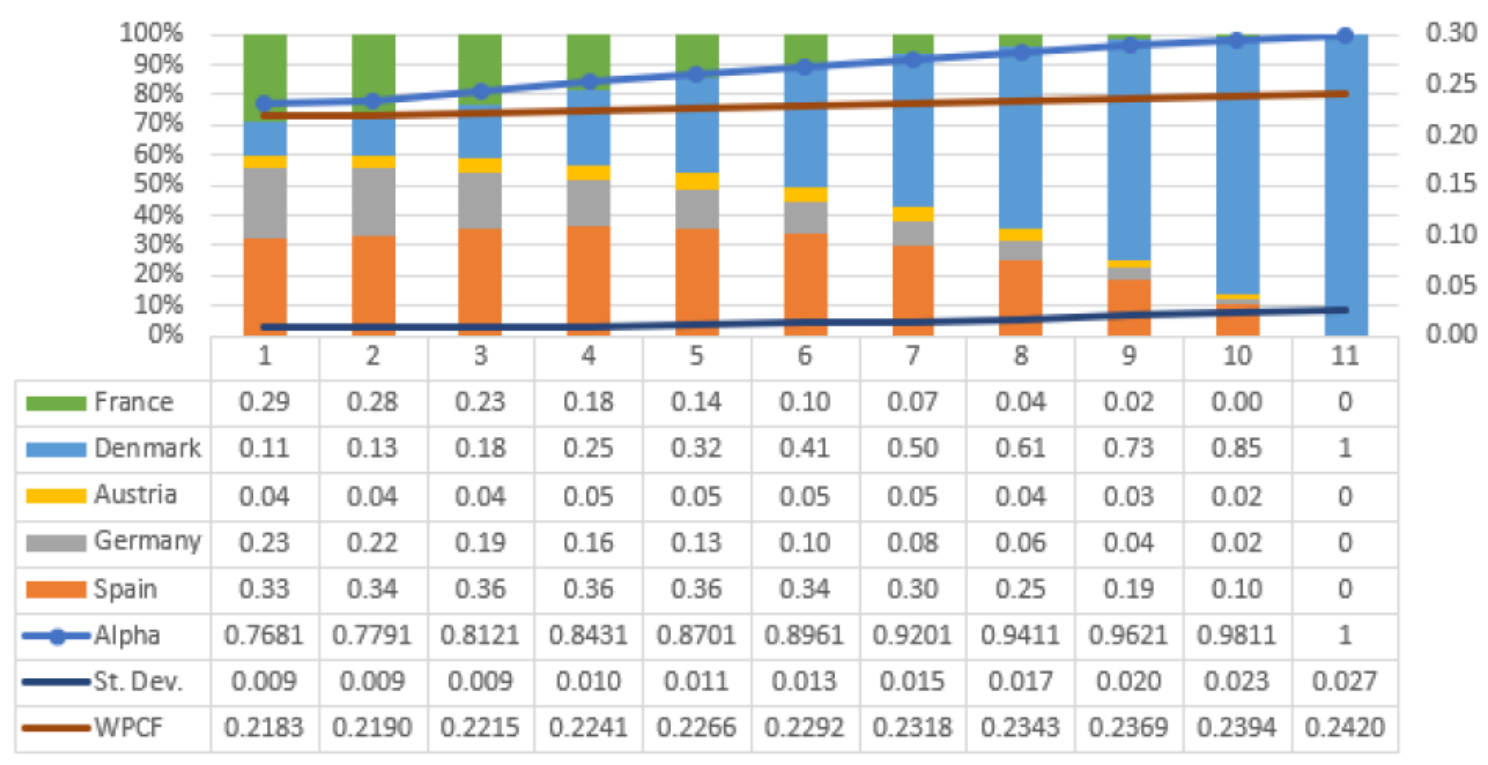

Figure 8. EF of PGP, Dn-Au-Sp-Fr-Gr

We obtain a very different EF from that obtained by Roques et al. (2010). For example, we find that the efficient portfolio that reach a WPCF of 0,2318 allocates the following weights to the five countries: Spain $30 \%$, Germany $8 \%$, Austria $5 \%$, France $7 \%$, and Denmark $50 \%$. On the other hand, Roques et al. (2010) find that, for the same level of WPCF, the efficient portfolio allocates the following weights to the five countries: Spain $54 \%$, Germany $0 \%$, Austria 6\%, France 10\%, and Denmark $31 \%$. The differences are noteworthy in the weights received by Denmark, Spain, and Germany. In our analysis, Germany receives a weight greater than zero while the weights received by Denmark and Spain are nearly switched between each other in the paper by Roques et al. (2010).

We find our results more coherent than those obtained by Roques et al. (2010). To see this, recall the fact that the expected return of the PGP is a convex sum of the expected returns of the different assets. Thus, as the WPCF of 0,2318 is closer to the expected return of Denmark and above the expected return of Spain, we expect that Denmark should receive a greater weight than Spain in the PGP. Which hold in our analysis as the grater weight received by Spain is $33 \%$.

When replicating the case of maximizing the wind-power contribution to the reliability of the system, we also find different results from those of Roques et al. (2010). Unlike these authors, we find that Germany receives a weight greater than zero and up to $15 \%$ in the PGP (This case will be provided upon request).

Notably, the difference in the results creates a bias in the design of policies in electricity generation by cross-country wind-power.

Roques et al. (2010) also find the EF of çonstrained"portfolios. These authors consider two types of constraints: 1)wind resource potential constraints, and 2) network limitations constraints. However, they do not provide the statistics of the returns for each scenario. The present methodology could be applied to replicate the results of such scenarios if the statistics of the returns were available.

\section{Final remarks and conclusions}

The present paper tackles the problem of the diversification of energy generation by providing a methodology to construct parametrically the EF of PGP for up to five assets. The methodology was applied to replicate 
and extend the results of the following three papers: Roques et al. (2008), Jain et al. (2014), and Roques et al. (2010). The present methodology has remarkable advantages:

1. It allows to conduct analysis with up to five assets together.

2. It allows to know the shares of the assets for any risk-return profile.

3. The EF could be limited by a maximum allowed risk of the PGP.

4. It provides more coherent results than those obtained in the existing literature.

5. It points out that there are optimal investment alternatives that have been denied by previous analysis.

6. It avoids biases in the design of investment policies in electricity generation.

Thus, the parametric formulation of PGP proved to be a powerful tool for power generation policy-makers or investors. In fact, it could be applied to portfolios of assets different than those of power-generation technologies.

From the structure of the paper, it would be straight forward to extend the methodology to obtaining the shares of assets in order to guarantee the minimal risk of PGP of more than five assets. However, the corresponding mathematical proofs must be performed to construct the EF of PGP parametrically of more than five assets.

Complete analysis relies on the assumption that the covariances of the returns amongst the different assets is zero. Depending on computational availability, future research could be extended to verify the actual effect of the correlations of the returns on the minimal risk of the portfolio.

\section{Referencias}

[1] Adams, R., Jamasb, T., 2016. Optimal Power Generation Portfolios with Renewables: An Application to the UK, Cambridge Working Papers in Economics 1646, Faculty of Economics, University of Cambridge. http://www.econ.cam.ac.uk/research-files/repec/cam/pdf/cwpe1646.pdf

[2] Allan, G., Eromenko, I., McGregor, P., Swales, K., 2011. The regional electricity generation mix in Scotland: A portfolio selection approach incorporating marine technologies. Energy Policy 39: 6-22. https://doi.org/10.1016/j.enpol.2010.08.028.

[3] Arnesano, M., Carlucci A. P., Laforgia D., 2012. Extension of portfolio theory application to energy planning problem — The Italian case. Energy 39: 112-114. doi:https://doi.org/10.1016/j.energy.2011.06.053

[4] Awerbuch, S., Berger, M., 2003. Applying portfolio Theory to EU Electricity Planning and Policy-Making. IEA/EET Working Paper. IEA. Paris. https://pdfs.semanticscholar.org/0ca8/8df704e4f08ebb458bcf8c4cc418f3eaa634.pdf?_ga=2.112372356.1645367726.15905203371249187651.1590520337

[5] Bhattacharya A., Kojima S., 2012. Power sector investment risk and renewable energy: A Japanese case study using portfolio risk optimization method. Energy Policy 40: 69-80. doi:https://doi.org/10.1016/j.enpol.2010.09.031

[6] Costa, O. L.V., Ribeiro, C. O., Rego, E. E., Stern, J. M., Parente, V., Kileber, S., 2017. Robust portfolio optimization for electricity planning: An application based on the Brazilian electricity mix. Energy Economics 64: 158-169. doi:https://doi.org/10.1016/j.eneco.2017.03.021

[7] Cunha, J., Ferreira, P., 2014. Designing electricity generation portfolios using the mean-variance approach, Int. J. Sustain. Energy Plan. Manag., 4: 17-30. doi:https://doi.org/10.5278/ijsepm.2014.4.3

[8] Delarue E., Jonghe C., Belmans R., D'haeseleer W., 2011. Applying portfolio theory to the electricity sector: Energy versus power. Energy Economics 33: 12-23. doi:https://doi.org/10.1016/j.eneco.2010.05.003 
[9] DeLlano-Paz, F., Calvo-Silvosa, A., Antelo, S. I., Soares, I., 2017. Energy planning and modern portfolio theory: A review. Renewable and Sustainable Energy Review 77: 636-651. doi:https://doi.org/10.1016/j.rser.2017.04.045

[10] Francés G. E., Marín-Quemada J.M., González E. S., 2013. RES and risk: Renewable energy's contribution to energy security. A portfolio based approach, Renewable and Sustainable Energy Reviews 26: 549-559. doi:https://doi.org/10.1016/j.rser.2013.06.015

[11] Freund, J. E., Miller, I, Miller, M., 2000. Estadística matemática con aplicaciones. Pearson educación.

[12] Gómez-Ríos, M., and Juárez-Luna, D. (2019). Cost of electric generation accounting for environmental externalities: Optimal mix of baseload technologies. Revista Mexicana de Economía y Finanzas Nueva Época REMEF, 14(3), 353-377. doi:https://doi.org/10.21919/remef.v14i3.308

[13] Jain, S., Roelofs, F., Oosterlee, C. W., 2014. Decision-support tool for assessing future nuclear reactor generation portfolios. Energy Economics 44: 99-112. doi:https://doi.org/10.1016/j.eneco.2014.03.021

[14] Jansen, J. C., Beurskens, L.W.M., Tilburg, X.V., 2006. Application of portfolio analysis to the Dutch generating mix. ECN report C-05-100. Energy research council of Netherlands. doi:https://publicaties.ecn.nl/PdfFetch.aspx?nr=ECN-C-05-100

[15] Losekann L., Marrero G. A., Ramos-Real F. J., Almeida E. L., 2013. Efficient power generating portfolio in Brazil: Conciliating cost, emissions and risk. Energy Policy 62: 301-314. doi:https://doi.org/10.1016/j.enpol.2013.07.049

[16] Markowitz H. M., 1952. Portfolio Selection, Journal of Finance, Vol. 7, pp 77-91. DOI: 10.2307/2975974

[17] Perea González, A., and Zavaleta Vazquez, O. (2020). Designing an optimal electricity supply portfolio based on the

[18] Markowitz model: The case of a user in Mexico. Contaduría y Administración, 65(1), 1-20. doi:http://dx.doi.org/10.22201/fca.24488410e.2019.1833

[19] Pinheiro Neto, D., Domingues, E. G., Coimbra, A. P., de Almeida, A. T., Alves, A. J., Calixto W. P., 2017. Portfolio optimization of renewable energy assets: hydro, wind, and photovoltaic energy in the regulated market in Brazil. Energy Economics 64: 238-250. doi:https://doi.org/10.1016/j.eneco.2017.03.020

[20] Roques, F. A., Hiroux, C., Saguan, M., 2010. Optimal wind power deployment in Europe - A portfolio approach, Energy Policy 38: 3245-3256. doi:https://doi.org/10.1016/j.enpol.2009.07.048

[21] Roques, F. A., Newbery, D. M., Nuttall, W. J., 2008. Fuel mix diversification incentives in liberalized electricity markets: A Mean-Variance Portfolio theory approach, Energy Economics, Volume 30, Issue 4: 1831-1849. doi:https://doi.org/10.1016/j.eneco.2007.11.008

[22] Roques, F. (2006). Power generation investments in liberalised markets: methodologies to capture risk, flexibility, and portfolio diversity. Économies et Sociétés, Série Economie de l'Energie 10 (Oct./Nov.), 1563-94.

[23] Vithayasrichareon, P., MacGill, I.F., Wen, F.S., 2010a. Electricity generation portfolio evaluation for highly uncertain and carbon constrained future electricity industries. IEEE Power and Energy Society General Meeting. doi:https://doi.org/10.1109/pes.2010.5589354

[24] Vithayasrichareon, P., MacGill, I.F., Wen, F., 2010b. Electricity Generation Portfolio Analysis for Coal, Gas and Nuclear Plant under Future Uncertainties. 4th IASTED Asian Conference on Power and Energy Systems. doi:https://doi.org/10.2316/p.2010.701-027

\section{Appendix}

[Proof of Proposition 1] The risk of the PGP of two technologies is given by $\sigma_{Y}=\sqrt{\alpha_{1}^{2} \sigma_{1}^{2}+\alpha_{2}^{2} \sigma_{2}^{2}}$. Assume that the expected return of technology 1 is the least risky. For $\sigma_{1,2}=0, \alpha_{i} \in[0,1]$ for $i=1,2$, and $\alpha_{1}+\alpha_{2}=1$. For tractability, the majority of the proof uses the variance of the PGP instead of its SD.

Proof of a) We need to find the shares of technologies 1 and 2 , given by $\left(\alpha_{1}, \alpha_{2}\right)$, which guarantee the minimum risk (variance) of the PGP. For tractability, take $\alpha_{2}=1-\alpha_{1}$. Then, the risk of the PGP is given by 
$\sigma_{Y}^{2}=\alpha_{1}^{2} \sigma_{1}^{2}+\left(1-\alpha_{1}\right)^{2} \sigma_{2}^{2}$. First, we find the critical point of the risk of the PGP. The First Order Conditions (FOC) are as follows:

$$
\partial \sigma_{Y}^{2} / \partial \alpha_{1}=2 \alpha_{1} \sigma_{1}^{2}+2\left(1-\alpha_{1}\right)(-1) \sigma_{2}^{2}=0
$$

From expression (5), we have

$$
\alpha_{1} \sigma_{1}^{2}+\alpha_{1} \sigma_{2}^{2}=\sigma_{2}^{2}
$$

which leads to

$$
\alpha_{1}^{*}=\sigma_{2}^{2} /\left[\sigma_{1}^{2}+\sigma_{2}^{2}\right],
$$

Thus, $\alpha_{2}^{*}=1-\alpha_{1}^{*}=\sigma_{1}^{2} /\left[\sigma_{1}^{2}+\sigma_{2}^{2}\right]$. The critical point of the risk of the PGP is given by shares

$$
\left(\alpha_{1}^{*}, \alpha_{2}^{*}\right)=\left(1 /\left[\sigma_{1}^{2}+\sigma_{2}^{2}\right]\right)\left(\sigma_{2}^{2}, \sigma_{1}^{2}\right),
$$

To verify that the risk of the PGP, $\sigma_{Y}^{2}$, has a minimum at the critical point $\left(\alpha_{1}^{*}, \alpha_{2}^{*}\right)$, we need the Second Order Conditions (SOC):

$$
\partial^{2} \sigma_{Y}^{2} / \partial \alpha_{1}^{2}=2\left[\sigma_{1}^{2}+\sigma_{2}^{2}\right]>0 .
$$

Thus, the risk of the PGP is a convex function of the shares of the two technologies, $\left(\alpha_{1}, \alpha_{2}\right)$. As a consequence, the risk of the PGP, $\sigma_{Y}^{2}$, has a global minimum when the shares of technologies 1 and 2 are given by expression (8).

Proof of $\mathbf{b})$ Thus, the minimal risk of the PGP, $\sigma_{Y}^{* 2}$, is given by

$$
\begin{gathered}
\sigma_{Y}^{* 2}=\left(1 /\left[\sigma_{1}^{2}+\sigma_{2}^{2}\right]^{2}\right)\left[\left(\sigma_{2}^{2}\right)^{2} \sigma_{1}^{2}+\left(\sigma_{1}^{2}\right)^{2} \sigma_{2}^{2}\right], \\
\sigma_{Y}^{* 2}=\left(\left[\sigma_{1}^{2} \sigma_{2}^{2}\right] /\left[\sigma_{1}^{2}+\sigma_{2}^{2}\right]^{2}\right)\left[\sigma_{2}^{2}+\sigma_{1}^{2}\right], \\
\sigma_{Y}^{* 2}=\left[\sigma_{1}^{2} \sigma_{2}^{2}\right] /\left[\sigma_{1}^{2}+\sigma_{2}^{2}\right]=\alpha_{1}^{*} \sigma_{1}^{2}<\sigma_{1}^{2},
\end{gathered}
$$

Then

$$
\sigma_{Y}^{*}<\sigma_{1}
$$

The minimal risk of the PGP is lower than the risk of the least risky technology.

[Proof of Proposition 2] Let $\sigma_{Y}=\sqrt{\alpha_{1}^{2} \sigma_{1}^{2}+\alpha_{2}^{2} \sigma_{2}^{2}}$ be the risk of the PGP of two technologies. From Proposition 3 , we know that the risk of the PGP, $\sigma_{Y}$, reaches its global minimum at point $\left(\alpha_{1}^{*}, \alpha_{2}^{*}\right)$. Assume that $\mu_{1} \geq \mu_{2}$. To obtain the parametric formulation of the EF, we express the risk of the PGP as follows:

$$
\sigma_{Y}^{2}=\alpha^{2} \sigma_{1}^{2}+(1-\alpha)^{2} \sigma_{2}^{2},
$$

for $\alpha \in[0,1]$. Note that when $\alpha=1$, then $\sigma_{Y}^{2}=\sigma_{1}^{2}$, the risk of the PGP equals the risk of technology 1 . This scenario ensures that technology 1, which has the greatest expected return, receives a share of $100 \%$. On the other hand, when $\alpha=0$, then $\sigma_{Y}^{2}=\sigma_{2}^{2}$, the risk of the PGP equals the risk of technology 2. The latter implies that technology 2, which has the lowest expected return, receives a share of $100 \%$. Thus, this formulation of the risk of the PGP allows to have portfolios assigning a share of $100 \%$ to the technologies with the greatest and least expected return. We need to ensure that expression (10) allows to reach the point $\left(\alpha_{1}^{*}, \alpha_{2}^{*}\right)$ where $\sigma_{Y}$ reaches its global minimum. Then, it should hold that

$$
\begin{gathered}
\alpha_{1}^{*}=\alpha, \\
\alpha_{2}^{*}=1-\alpha,
\end{gathered}
$$

Expressions (11) and (12) lead to the fact that the shares of technologies 1 and 2 in the risk of the PGPs are given by

$$
\begin{gathered}
\alpha_{1}=\alpha, \\
\alpha_{2}=1-\alpha,
\end{gathered}
$$


Then, from expression (10) the risk of the PGP is given by

$$
\sigma_{Y}^{2}=\alpha^{2} \sigma_{1}^{2}+(1-\alpha)^{2} \sigma_{2}^{2},
$$

From expressions (11) and (13), the PGP with lowest risk is given by

$$
\alpha=\alpha_{1}^{*} .
$$

Now we need to find the PGP with the greatest expected return. The expected return of the PGP is given by:

$$
\mu_{Y}=\alpha_{1} \mu_{1}+\alpha_{2} \mu_{2},
$$

substituting expressions (13) and (14) in expression (17) leads to

$$
\mu_{Y}=\alpha \mu_{1}+(1-\alpha) \mu_{2},
$$

it is straightforward to obtain that

$$
d \mu_{Y} / d \alpha=\mu_{1}-\mu_{2} \geq 0,
$$

because of the assumption that $\mu_{1} \geq \mu_{2}$. Then, the PGP reaches its maximum expected return when $\alpha=1, \mu_{Y}=\mu_{1}$, and $\sigma_{Y}^{2}=\sigma_{1}^{2}$. However, there could be an alternative criterion to choose the maximal expected return of the PGP. For example, if the expected return of technology 1 is the least risky, then, the criterion could be to choose $\alpha^{d f}$, so that $\sigma_{Y}^{2}\left(\alpha^{d f}\right)=\sigma_{1}^{2}$; in this case, $\alpha^{d f}<1$. Then, the corresponding maximal expected return of the PGP is given by $\mu\left(\alpha^{d f}\right)$. Thus, the risk of the efficient portfolios is obtained by the shares of technologies 1 and 2, given by expressions (13) and (14) for $\alpha_{1}^{*} \leq \alpha \leq \alpha^{d f}$. As a consequence, the risk of the PGP in the EF is given by expression (15). The maximal expected return for each corresponding level of risk is given by expression (18). Note that $\mu\left(\alpha^{d f}\right) \leq \mu_{1}$ and $\sigma\left(\alpha^{d f}\right) \leq \sigma_{1}$.

[Proof of Proposition 3] The risk of the PGP of three technologies is given by $\sigma_{Y}=\sqrt{\sum_{i=1}^{3} \alpha_{i}^{2} \sigma_{i}^{2}}$. Assume that the expected return of technology 1 is the least risky. For $\sigma_{1,2}=\sigma_{1,3}=\sigma_{2,3}=0, \alpha_{i} \in[0,1]$ for $i=1,2,3$, and $\sum_{i=1}^{3} \alpha_{i}=1$. For tractability, the majority of the proof uses the variance of the PGP instead of its SD.

Proof of a) We need to find the shares of technologies 1, 2, and 3, given by $\left(\alpha_{1}, \alpha_{2}, \alpha_{3}\right)$, which ensures the minimum risk (variance) of the PGP. For tractability, take $\alpha_{3}=1-\alpha_{1}-\alpha_{2}$. Then, the risk of the PGP is given by $\sigma_{Y}^{2}=\alpha_{1}^{2} \sigma_{1}^{2}+\alpha_{2}^{2} \sigma_{2}^{2}+\left(1-\alpha_{1}-\alpha_{2}\right)^{2} \sigma_{3}^{2}$. First, we find the critical point of the risk of the PGP. The FOC are the following:

$$
\begin{aligned}
& \partial \sigma_{Y}^{2} / \partial \alpha_{1}=2 \alpha_{1} \sigma_{1}^{2}+2\left(1-\alpha_{1}-\alpha_{2}\right)(-1) \sigma_{3}^{2}=0, \\
& \partial \sigma_{Y}^{2} / \partial \alpha_{2}=2 \alpha_{2} \sigma_{2}^{2}+2\left(1-\alpha_{1}-\alpha_{2}\right)(-1) \sigma_{3}^{2}=0,
\end{aligned}
$$

from expression (19), we have

$$
\alpha_{1}\left[\sigma_{1}^{2}+\sigma_{3}^{2}\right]+\alpha_{2} \sigma_{3}^{2}=\sigma_{3}^{2}
$$

from expression (20), we have

$$
\alpha_{1} \sigma_{3}^{2}+\alpha_{2}\left[\sigma_{2}^{2}+\sigma_{3}^{2}\right]=\sigma_{3}^{2} .
$$

Expressions (21) and (22) lead to the following system of equations

$$
\left[\begin{array}{cc}
\sigma_{1}^{2}+\sigma_{3}^{2} & \sigma_{3}^{2} \\
\sigma_{3}^{2} & \sigma_{2}^{2}+\sigma_{3}^{2}
\end{array}\right]\left[\begin{array}{l}
\alpha_{1} \\
\alpha_{2}
\end{array}\right]=\left[\begin{array}{l}
\sigma_{3}^{2} \\
\sigma_{3}^{2}
\end{array}\right]
$$

Calculating the inverse of matrix $A_{3}=\left[\begin{array}{cc}\sigma_{1}^{2}+\sigma_{3}^{2} & \sigma_{3}^{2} \\ \sigma_{3}^{2} & \sigma_{2}^{2}+\sigma_{3}^{2}\end{array}\right]$ we end up with

$$
\left[\begin{array}{c}
\alpha_{1}^{*} \\
\alpha_{2}^{*}
\end{array}\right]=\frac{1}{\left|A_{3}\right|}\left[\begin{array}{cc}
\sigma_{2}^{2}+\sigma_{3}^{2} & -\sigma_{3}^{2} \\
-\sigma_{3}^{2} & \sigma_{1}^{2}+\sigma_{3}^{2}
\end{array}\right]\left[\begin{array}{c}
\sigma_{3}^{2} \\
\sigma_{3}^{2}
\end{array}\right] \text {, }
$$


where $\left|A_{3}\right|=\sigma_{1}^{2} \sigma_{2}^{2}+\sigma_{1}^{2} \sigma_{3}^{2}+\sigma_{2}^{2} \sigma_{3}^{2}$, leading to the result

$$
\left[\begin{array}{c}
\alpha_{1}^{*} \\
\alpha_{2}^{*}
\end{array}\right]=\frac{1}{\left|A_{3}\right|}\left[\begin{array}{c}
\sigma_{2}^{2} \sigma_{3}^{2} \\
\sigma_{1}^{2} \sigma_{3}^{2}
\end{array}\right] .
$$

Then, $\alpha_{3}^{*}=1-\alpha_{1}^{*}-\alpha_{2}^{*}=\sigma_{1}^{2} \sigma_{2}^{2} /\left|A_{3}\right|$. The critical point of the risk of the PGP is given by shares

$$
\left(\alpha_{1}^{*}, \alpha_{2}^{*}, \alpha_{3}^{*}\right)=\left[1 /\left|A_{3}\right|\right]\left(\sigma_{2}^{2} \sigma_{3}^{2}, \sigma_{1}^{2} \sigma_{3}^{2}, \sigma_{1}^{2} \sigma_{2}^{2}\right) .
$$

To verify that the risk of the PGP, $\sigma_{Y}^{2}$, has a minimum at the critical point $\left(\alpha_{1}^{*}, \alpha_{2}^{*}, \alpha_{3}^{*}\right)$, we need the SOC. The Hessian matrix is given as follows:

$$
H=2\left[\begin{array}{cc}
\sigma_{1}^{2}+\sigma_{3}^{2} & \sigma_{3}^{2} \\
\sigma_{3}^{2} & \sigma_{2}^{2}+\sigma_{3}^{2}
\end{array}\right]
$$

Following the criteria of the leading principal minors of the Hessian matrix, we have

$$
\begin{gathered}
H_{1}=2\left(\sigma_{1}^{2}+\sigma_{3}^{2}\right)>0 . \\
H_{2}=2\left|A_{3}\right|=2\left(\sigma_{1}^{2} \sigma_{2}^{2}+\sigma_{1}^{2} \sigma_{3}^{2}+\sigma_{2}^{2} \sigma_{3}^{2}\right)>0 .
\end{gathered}
$$

The two leading principal minors of the Hessian matrix are positive for any $\left(\alpha_{1}, \alpha_{2}, \alpha_{3}\right)$. Thus, the risk of the PGP is a convex function of the shares of the three technologies, $\left(\alpha_{1}, \alpha_{2}, \alpha_{3}\right)$. As a consequence, the risk of the PGP, $\sigma_{Y}^{2}$, has a global minimum when the shares of technologies 1,2 , and 3 are given by expression (26).

Proof of $\mathbf{b})$ Thus, the minimal risk of the PGP, $\sigma_{Y}^{* 2}$, is given by

$$
\begin{gathered}
\sigma_{Y}^{* 2}=\left(1 /\left|A_{3}\right|^{2}\right)\left[\left(\sigma_{2}^{2} \sigma_{3}^{2}\right)^{2} \sigma_{1}^{2}+\left(\sigma_{1}^{2} \sigma_{3}^{2}\right)^{2} \sigma_{2}^{2}+\left(\sigma_{1}^{2} \sigma_{2}^{2}\right)^{2} \sigma_{3}^{2}\right] \\
\sigma_{Y}^{* 2}=\left(\left[\sigma_{1}^{2} \sigma_{2}^{2} \sigma_{3}^{2}\right] /\left|A_{3}\right|^{2}\right)\left[\sigma_{1}^{2} \sigma_{2}^{2}+\sigma_{1}^{2} \sigma_{3}^{2}+\sigma_{2}^{2} \sigma_{3}^{2}\right] \\
\sigma_{Y}^{* 2}=\left[\sigma_{1}^{2} \sigma_{2}^{2} \sigma_{3}^{2}\right] /\left|A_{3}\right|=\alpha_{1}^{*} \sigma_{1}^{2}<\sigma_{1}^{2}
\end{gathered}
$$

Then

$$
\sigma_{Y}^{*}<\sigma_{1}
$$

The minimal risk of the PGP is lower than the risk of the least risky technology.

[Proof of Proposition 4] Let $\sigma_{Y}=\sqrt{\sum_{i=1}^{3} \alpha_{i}^{2} \sigma_{i}^{2}}$ be the risk of the PGP of three technologies. From Proposition 5, we know that the risk of the PGP, $\sigma_{Y}$, reaches its global minimum at point $\left(\alpha_{1}^{*}, \alpha_{2}^{*}, \alpha_{3}^{*}\right)$. Assume that $\mu_{1} \geq \mu_{2} \geq \mu_{3}$. To obtain the parametric formulation of the EF, we express the risk of the PGP as follows:

$$
\begin{aligned}
& \sigma_{Y}^{2}=\alpha^{2}\left(\beta^{2} \sigma_{1}^{2}+(1-\beta)^{2} \sigma_{2}^{2}\right)+(1-\alpha)^{2} \sigma_{3}^{2}, \\
& \sigma_{Y}^{2}=\alpha^{2} \beta^{2} \sigma_{1}^{2}+\alpha^{2}(1-\beta)^{2} \sigma_{2}^{2}+(1-\alpha)^{2} \sigma_{3}^{2},
\end{aligned}
$$

for $\alpha, \beta \in[0,1]$. Note that when $\alpha=\beta=1$, then $\sigma_{Y}^{2}=\sigma_{1}^{2}$, the risk of the PGP equals the risk of technology 1. This scenario implies that technology 1 , which has the greatest expected return, receives a share of $100 \%$. On the other hand, when $\alpha=0$, then $\sigma_{Y}^{2}=\sigma_{3}^{2}$, the risk of the PGP equals the risk of technology 3. Then, technology 3, which has the lowest expected return, receives a share of $100 \%$. Thus, this formulation of the risk of the PGP allows to have portfolios that assign a share of $100 \%$ to the technologies with the greatest and least expected return. We need to ensure that expression (28) allows to reach the point $\left(\alpha_{1}^{*}, \alpha_{2}^{*}, \alpha_{3}^{*}\right)$, where $\sigma_{Y}$ reaches its global minimum. Then, it should hold that

$$
\begin{gathered}
\alpha_{1}^{*}=\alpha \beta, \\
\alpha_{2}^{*}=\alpha(1-\beta), \\
\alpha_{3}^{*}=(1-\alpha),
\end{gathered}
$$


from expression (29)

$$
\alpha=\alpha_{1}^{*} / \beta
$$

substituting expression (32) in expression (30) leads to

$$
\beta=\alpha_{1}^{*} /\left[\alpha_{1}^{*}+\alpha_{2}^{*}\right]
$$

substituting expression (33) in expression (32) leads to

$$
\alpha=\alpha_{1}^{*}+\alpha_{2}^{*} .
$$

To ensure that $\beta \in[0,1]$ for $\alpha \in[0,1]$, assume that

$$
\beta=\beta(\alpha)=\alpha^{x}
$$

Then, substituting expressions (33) and (34) in expression (35) we have

$$
\alpha_{1}^{*} /\left[\alpha_{1}^{*}+\alpha_{2}^{*}\right]=\left(\alpha_{1}^{*}+\alpha_{2}^{*}\right)^{x} .
$$

which leads to

$$
x=\left(\ln \left[\alpha_{1}^{*} /\left(\alpha_{1}^{*}+\alpha_{2}^{*}\right)\right]\right) /\left(\ln \left[\alpha_{1}^{*}+\alpha_{2}^{*}\right]\right) .
$$

Substituting expression (35) in expressions (29), (30), and (31) leads to the fact that the shares of technologies 1,2 and 3 in the risk of the PGP are given by the following expressions:

$$
\begin{gathered}
\alpha_{1}=\alpha \beta=\alpha^{1+x}, \\
\alpha_{2}=\alpha(1-\beta)=\alpha-\alpha^{1+x}, \\
\alpha_{3}=1-\alpha .
\end{gathered}
$$

Then, substituting expressions (37), (38), and (39) into (28) leads to the risk of the PGP:

$$
\sigma_{Y}^{2}=\left[\alpha^{1+x}\right]^{2} \sigma_{1}^{2}+\left[\alpha-\alpha^{1+x}\right]^{2} \sigma_{2}^{2}+[1-\alpha]^{2} \sigma_{3}^{2} .
$$

From expressions (29) and (37), the PGP with lowest risk is given when

$$
\alpha=\left[\alpha_{1}^{*}\right]^{\frac{1}{1+x}} .
$$

Now we need to find the PGP with the greatest expected return. The expected return of the PGP is given by:

$$
\mu_{Y}=\alpha_{1} \mu_{1}+\alpha_{2} \mu_{2}+\alpha_{3} \mu_{3}
$$

substituting expressions (37), (38), and (39) in expression (42) leads to

$$
\mu_{Y}=\alpha^{1+x} \mu_{1}+\left(\alpha-\alpha^{1+x}\right) \mu_{2}+(1-\alpha) \mu_{3},
$$

It is straightforward to obtain that

$$
d \mu_{Y} / d \alpha=[1+x] \alpha^{x}\left[\mu_{1}-\mu_{2}\right]+\mu_{2}-\mu_{3} \geq 0,
$$

because of the assumption that $\mu_{1} \geq \mu_{2} \geq \mu_{3}$. Then, the PGP reaches its maximum expected return when $\alpha=1, \mu_{Y}=\mu_{1}$ and $\sigma_{Y}^{2}=\sigma_{1}^{2}$. However, there could be an alternative criterion to choose the maximal expected return of the PGP. For example, if the expected return of technology 1 is the least risky, then, the criterion could be to choose $\alpha^{d f}$, so that $\sigma_{Y}^{2}\left(\alpha^{d f}\right)=\sigma_{1}^{2}$, in this case $\alpha^{d f}<1$. Then, the corresponding maximal expected return of the PGP is given by $\mu\left(\alpha^{d f}\right)$. Thus, the risk of the efficient portfolios is obtained by the shares of technologies 1,2 , and 3, given by expressions (37), (38), and (39) for $\left[\alpha_{1}^{*}\right]^{\frac{1}{1+x}} \leq \alpha \leq$ $\alpha^{d f}$. As a consequence, the risk of the PGP in the EF is given by expression (40). The maximal expected rerun for each corresponding level of risk is given by expression (43). Note that $\mu\left(\alpha^{d f}\right) \leq \mu_{1}$ and $\sigma\left(\alpha^{d f}\right) \leq \sigma_{1}$. 
[Proof of Proposition 5] We conducted the proof for PGP of three technologies. However, it is straightforward to extend the proof to PGP of more than three technologies. Consider three-power generation technologies with corresponding risks $\sigma_{1}^{2}, \sigma_{2}^{2}$, and $\sigma_{3}^{2}$. The minimal risk reached by a PGP consisting of technology 1 is $\sigma_{Y_{1}}^{*}=\sigma_{1}$. From proposition $3 \mathrm{~b}$, the minimal risk reached by a PGP of two technologies is $\sigma_{Y_{2}}^{*}=\sqrt{\left[\sigma_{1}^{2} \sigma_{2}^{2}\right] /\left[\sigma_{1}^{2}+\sigma_{2}^{2}\right]}$. From proposition 5b, the minimal risk reached by a PGP of three technologies is $\sigma_{Y_{3}}^{*}=\sqrt{\left[\sigma_{1}^{2} \sigma_{2}^{2} \sigma_{3}^{2}\right] /\left|A_{3}\right|}$, where $\left|A_{3}\right|=\sigma_{1}^{2} \sigma_{2}^{2}+\sigma_{1}^{2} \sigma_{3}^{2}+\sigma_{2}^{2} \sigma_{3}^{2}$. We need to prove that $\sigma_{Y_{1}}^{*}>\sigma_{Y_{2}}^{*}>\sigma_{Y_{3}}^{*}$.

a) First, we verify whether $\sigma_{Y_{1}}^{*}>\sigma_{Y_{2}}^{*}$ holds:

$$
\begin{gathered}
\sigma_{1}>\sqrt{\left[\sigma_{1}^{2} \sigma_{2}^{2}\right] /\left[\sigma_{1}^{2}+\sigma_{2}^{2}\right]}, \\
\sigma_{1}^{2}>\left[\sigma_{1}^{2} \sigma_{2}^{2}\right] /\left[\sigma_{1}^{2}+\sigma_{2}^{2}\right], \\
\sigma_{1}^{4}+\sigma_{1}^{2} \sigma_{2}^{2}>\sigma_{1}^{2} \sigma_{2}^{2}, \\
\Rightarrow \sigma_{1}^{4}>0 .
\end{gathered}
$$

Then, it holds that $\sigma_{Y_{1}}^{*}>\sigma_{Y_{2}}^{*}$.

b) Second, we verify whether $\sigma_{Y_{2}}^{*}>\sigma_{Y_{3}}^{*}$ holds:

$$
\begin{gathered}
\sqrt{\left[\sigma_{1}^{2} \sigma_{2}^{2}\right] /\left[\sigma_{1}^{2}+\sigma_{2}^{2}\right]}>\sqrt{\left[\sigma_{1}^{2} \sigma_{2}^{2} \sigma_{3}^{2}\right] /\left|A_{3}\right|}, \\
{\left[\sigma_{1}^{2} \sigma_{2}^{2}\right] /\left[\sigma_{1}^{2}+\sigma_{2}^{2}\right]>\left[\sigma_{1}^{2} \sigma_{2}^{2} \sigma_{3}^{2}\right] /\left|A_{3}\right|,} \\
\sigma_{1}^{4} \sigma_{2}^{4}+\sigma_{1}^{4} \sigma_{2}^{2} \sigma_{3}^{2}+\sigma_{1}^{2} \sigma_{2}^{4} \sigma_{3}^{2}>\sigma_{1}^{4} \sigma_{2}^{2} \sigma_{3}^{2}+\sigma_{1}^{2} \sigma_{2}^{4} \sigma_{3}^{2}, \\
\Rightarrow \sigma_{1}^{4} \sigma_{2}^{4}>0 .
\end{gathered}
$$

Then, it holds that $\sigma_{Y_{2}}^{*}>\sigma_{Y_{3}}^{*}$. It follows that $\sigma_{Y_{n}}^{*}<\sigma_{Y_{k}}^{*}$ for $k<n$.

[Proof of Proposition 6] The risk of the PGP of four nuclear reactors is given by $\sigma_{Y}=\sqrt{\sum_{i=1}^{4} \alpha_{i}^{2} \sigma_{i}^{2}}$. Assume that the expected return of reactor 4 is the least risky. If $\sigma_{i, j}=0$, for any values $i$ and $j$, from 1 to 4 , such that $i<j$. If $\alpha_{i} \in[0,1]$ for $i=1,2,3,4$, and $\sum_{i=1}^{4} \alpha_{i}=1$. For tractability, the majority of the proof uses the variance of the PGP instead of its SD.

Proof of a) We need to find the shares of reactors 1, 2, 3, and 4, given by $\left(\alpha_{1}, \alpha_{2}, \alpha_{3}, \alpha_{4}\right)$, which ensures the minimum risk (variance) of PGP. For tractability, take $\alpha_{4}=1-\alpha_{1}-\alpha_{2}-\alpha_{3}$. Then, the risk of the PGP is given by $\sigma_{Y}^{2}=\alpha_{1}^{2} \sigma_{1}^{2}+\alpha_{2}^{2} \sigma_{2}^{2}+\alpha_{3}^{2} \sigma_{3}^{2}+\left(1-\alpha_{1}-\alpha_{2}-\alpha_{3}\right)^{2} \sigma_{4}^{2}$. First, we find the critical point of the PGP. The FOC are the following:

$$
\begin{aligned}
& \partial \sigma_{Y}^{2} / \partial \alpha_{1}=2 \alpha_{1} \sigma_{1}^{2}+2\left(1-\alpha_{1}-\alpha_{2}-\alpha_{3}\right)(-1) \sigma_{4}^{2}=0, \\
& \partial \sigma_{Y}^{2} / \partial \alpha_{2}=2 \alpha_{2} \sigma_{2}^{2}+2\left(1-\alpha_{1}-\alpha_{2}-\alpha_{3}\right)(-1) \sigma_{4}^{2}=0, \\
& \partial \sigma_{Y}^{2} / \partial \alpha_{3}=2 \alpha_{3} \sigma_{3}^{2}+2\left(1-\alpha_{1}-\alpha_{2}-\alpha_{3}\right)(-1) \sigma_{4}^{2}=0,
\end{aligned}
$$

from expression (44), we have

$$
\alpha_{1}\left[\sigma_{1}^{2}+\sigma_{4}^{2}\right]+\alpha_{2} \sigma_{4}^{2}+\alpha_{3} \sigma_{4}^{2}=\sigma_{4}^{2},
$$

from expression (45), we have

$$
\alpha_{1} \sigma_{4}^{2}+\alpha_{2}\left[\sigma_{2}^{2}+\sigma_{4}^{2}\right]+\alpha_{3} \sigma_{4}^{2}=\sigma_{4}^{2},
$$

from expression (46), we have

$$
\alpha_{1} \sigma_{4}^{2}+\alpha_{2} \sigma_{4}^{2}+\alpha_{3}\left[\sigma_{3}^{2}+\sigma_{4}^{2}\right]=\sigma_{4}^{2} .
$$

Expressions (47), (48), and (49) lead to the following system of equations

$$
\left[\begin{array}{ccc}
\sigma_{1}^{2}+\sigma_{4}^{2} & \sigma_{4}^{2} & \sigma_{4}^{2} \\
\sigma_{4}^{2} & \sigma_{2}^{2}+\sigma_{4}^{2} & \sigma_{4}^{2} \\
\sigma_{4}^{2} & \sigma_{4}^{2} & \sigma_{3}^{2}+\sigma_{4}^{2}
\end{array}\right]\left[\begin{array}{c}
\alpha_{1} \\
\alpha_{2} \\
\alpha_{3}
\end{array}\right]=\left[\begin{array}{c}
\sigma_{4}^{2} \\
\sigma_{4}^{2} \\
\sigma_{4}^{2}
\end{array}\right],
$$


Calculating the inverse of matrix $A_{4}=\left[\begin{array}{ccc}\sigma_{1}^{2}+\sigma_{4}^{2} & \sigma_{4}^{2} & \sigma_{4}^{2} \\ \sigma_{4}^{2} & \sigma_{2}^{2}+\sigma_{4}^{2} & \sigma_{4}^{2} \\ \sigma_{4}^{2} & \sigma_{4}^{2} & \sigma_{3}^{2}+\sigma_{4}^{2}\end{array}\right]$ we end up with

$$
\left[\begin{array}{c}
\alpha_{1}^{*} \\
\alpha_{2}^{*} \\
\alpha_{3}^{*}
\end{array}\right]=\frac{1}{\left|A_{4}\right|}\left[\begin{array}{ccc}
\sigma_{2}^{2} \sigma_{3}^{2}+\sigma_{2}^{2} \sigma_{4}^{2}+\sigma_{3}^{2} \sigma_{4}^{2} & -\sigma_{3}^{2} \sigma_{4}^{2} & -\sigma_{2}^{2} \sigma_{4}^{2} \\
-\sigma_{3}^{2} \sigma_{4}^{2} & \sigma_{1}^{2} \sigma_{3}^{2}+\sigma_{1}^{2} \sigma_{4}^{2}+\sigma_{3}^{2} \sigma_{4}^{2} & -\sigma_{1}^{2} \sigma_{4}^{2} \\
-\sigma_{2}^{2} \sigma_{4}^{2} & -\sigma_{1}^{2} \sigma_{4}^{2} & \sigma_{1}^{2} \sigma_{2}^{2}+\sigma_{1}^{2} \sigma_{4}^{2}+\sigma_{2}^{2} \sigma_{4}^{2}
\end{array}\right]\left[\begin{array}{c}
\sigma_{4}^{2} \\
\sigma_{4}^{2} \\
\sigma_{4}^{2}
\end{array}\right],
$$

where $\left|A_{4}\right|=\sigma_{1}^{2} \sigma_{2}^{2} \sigma_{3}^{2}+\sigma_{1}^{2} \sigma_{2}^{2} \sigma_{4}^{2}+\sigma_{1}^{2} \sigma_{3}^{2} \sigma_{4}^{2}+\sigma_{2}^{2} \sigma_{3}^{2} \sigma_{4}^{2}$. The solution of the system of equations is

$$
\left[\begin{array}{l}
\alpha_{1}^{*} \\
\alpha_{2}^{*} \\
\alpha_{3}^{*}
\end{array}\right]=\frac{1}{\left|A_{4}\right|}\left[\begin{array}{l}
\sigma_{2}^{2} \sigma_{3}^{2} \sigma_{4}^{2} \\
\sigma_{1}^{2} \sigma_{3}^{2} \sigma_{4}^{2} \\
\sigma_{1}^{2} \sigma_{2}^{2} \sigma_{4}^{2}
\end{array}\right]
$$

Then, $\alpha_{4}^{*}=1-\alpha_{1}^{*}-\alpha_{2}^{*}-\alpha_{3}^{*}=\sigma_{1}^{2} \sigma_{2}^{2} \sigma_{3}^{2} /\left|A_{4}\right|$. The critical point of the risk of the PGP is given by shares

$$
\left(\alpha_{1}^{*}, \alpha_{2}^{*}, \alpha_{3}^{*}, \alpha_{4}^{*}\right)=\left[1 /\left|A_{4}\right|\right]\left(\sigma_{2}^{2} \sigma_{3}^{2} \sigma_{4}^{2}, \sigma_{1}^{2} \sigma_{3}^{2} \sigma_{4}^{2}, \sigma_{1}^{2} \sigma_{2}^{2} \sigma_{4}^{2}, \sigma_{1}^{2} \sigma_{2}^{2} \sigma_{3}^{2}\right) .
$$

To verify that the risk of the PGP, $\sigma_{Y}^{2}$, has a minimum at the critical point $\left(\alpha_{1}^{*}, \alpha_{2}^{*}, \alpha_{3}^{*}, \alpha_{4}^{*}\right)$, we need the SOC. The Hessian matrix is given as follows:

$$
H=2\left[\begin{array}{ccc}
\sigma_{1}^{2}+\sigma_{4}^{2} & \sigma_{4}^{2} & \sigma_{4}^{2} \\
\sigma_{4}^{2} & \sigma_{2}^{2}+\sigma_{4}^{2} & \sigma_{4}^{2} \\
\sigma_{4}^{2} & \sigma_{4}^{2} & \sigma_{3}^{2}+\sigma_{4}^{2}
\end{array}\right]
$$

Following the criteria of the leading principal minors of the Hessian matrix, we have

$$
\begin{gathered}
H_{1}=2\left(\sigma_{1}^{2}+\sigma_{4}^{2}\right)>0 \\
H_{2}=2\left|\begin{array}{cc}
\sigma_{1}^{2}+\sigma_{4}^{2} & \sigma_{4}^{2} \\
\sigma_{4}^{2} & \sigma_{2}^{2}+\sigma_{4}^{2}
\end{array}\right|=2\left(\sigma_{1}^{2} \sigma_{2}^{2}+\sigma_{1}^{2} \sigma_{4}^{2}+\sigma_{2}^{2} \sigma_{4}^{2}\right)>0 \\
H_{3}=2\left|A_{4}\right|>0 .
\end{gathered}
$$

The three leading principal minors of the Hessian matrix are positive for any $\left(\alpha_{1}, \alpha_{2}, \alpha_{3}, \alpha_{4}\right)$. Then, the risk of the PGP is a convex function of the shares of the four reactors, $\left(\alpha_{1}, \alpha_{2}, \alpha_{3}, \alpha_{4}\right)$. As a consequence, the risk of the PGP, $\sigma_{Y}^{2}$, has a global minimum when the shares of reactors $1,2,3$, and 4 are given by expression (53).

Proof of b) Then, the minimal risk of the PGP portfolio, $\sigma_{Y}^{* 2}$, is given by

$$
\begin{gathered}
\sigma_{Y}^{* 2}=\left(1 /\left|A_{4}\right|^{2}\right)\left[\left(\sigma_{2}^{2} \sigma_{3}^{2} \sigma_{4}^{2}\right)^{2} \sigma_{1}^{2}+\left(\sigma_{1}^{2} \sigma_{3}^{2} \sigma_{4}^{2}\right)^{2} \sigma_{2}^{2}+\left(\sigma_{1}^{2} \sigma_{2}^{2} \sigma_{4}^{2}\right)^{2} \sigma_{3}^{2}+\left(\sigma_{1}^{2} \sigma_{2}^{2} \sigma_{3}^{2}\right)^{2} \sigma_{4}^{2}\right] \\
\sigma_{Y}^{* 2}=\left(\left[\sigma_{1}^{2} \sigma_{2}^{2} \sigma_{3}^{2} \sigma_{4}^{2}\right] /\left|A_{4}\right|^{2}\right)\left[\sigma_{2}^{2} \sigma_{3}^{2} \sigma_{4}^{2}+\sigma_{1}^{2} \sigma_{3}^{2} \sigma_{4}^{2}+\sigma_{1}^{2} \sigma_{2}^{2} \sigma_{4}^{2}+\sigma_{1}^{2} \sigma_{2}^{2} \sigma_{3}^{2}\right] \\
\sigma_{Y}^{* 2}=\sigma_{1}^{2} \sigma_{2}^{2} \sigma_{3}^{2} \sigma_{4}^{2} /\left|A_{4}\right|=\alpha_{4}^{*} \sigma_{4}^{2}<\sigma_{4}^{2}
\end{gathered}
$$

Then

$$
\sigma_{Y}^{*}<\sigma_{4}
$$

The minimal risk of the PGP is lower than the risk of the less risky reactor.

[Proof of Proposition 7] Let $\sigma_{Y}=\sqrt{\sum_{i=1}^{4} \alpha_{i}^{2} \sigma_{i}^{2}}$ the risk of the PGP of four reactors. From Proposition 8 , we know that the risk of the PGP, $\sigma_{Y}$, reaches its global minimum at point $\left(\alpha_{1}^{*}, \alpha_{2}^{*}, \alpha_{3}^{*}, \alpha_{4}^{*}\right)$. Assume that $\mu_{1} \geq \mu_{2} \geq \mu_{3} \geq \mu_{4}$. To obtain the parametric formulation of the EF, we express the risk of the PGP as follows:

$$
\begin{gathered}
\sigma_{Y}^{2}=\alpha^{2}\left[\beta^{2} \sigma_{1}^{2}+(1-\beta)^{2} \sigma_{2}^{2}\right]+(1-\alpha)^{2}\left[\gamma^{2} \sigma_{3}^{2}+(1-\gamma)^{2} \sigma_{4}^{2}\right], \\
\sigma_{Y}^{2}=\alpha^{2} \beta^{2} \sigma_{1}^{2}+\alpha^{2}(1-\beta)^{2} \sigma_{2}^{2}+(1-\alpha)^{2} \gamma^{2} \sigma_{3}^{2}+(1-\alpha)^{2}(1-\gamma)^{2} \sigma_{4}^{2}
\end{gathered}
$$


for $\alpha, \beta, \gamma \in[0,1]$. Note that when $\alpha=\beta=1$, then $\sigma_{Y}^{2}=\sigma_{1}^{2}$, the risk of the PGP equals the risk of reactor 1. This scenario implies that reactor 1 , which has the greatest expected return, receives a share of $100 \%$. On the other hand, when $\alpha=\gamma=0$, then $\sigma_{Y}^{2}=\sigma_{4}^{2}$, the risk of the PGP equals the risk of reactor 4 . Then, reactor 4, which has the least expected return, receives a share of $100 \%$. Thus, this formulation of the risk of the PGP allows to have portfolios that assign a share of $100 \%$ to the reactors with the greatest and lowest expected return. We need to be sure that expression (56) allows to reach the point $\left(\alpha_{1}^{*}, \alpha_{2}^{*}, \alpha_{3}^{*}, \alpha_{4}^{*}\right)$, where $\sigma_{Y}$ reaches its global minimum. Then, it should holds that

$$
\begin{gathered}
\alpha_{1}^{*}=\alpha \beta, \\
\alpha_{2}^{*}=\alpha(1-\beta), \\
\alpha_{3}^{*}=(1-\alpha) \gamma, \\
\alpha_{4}^{*}=(1-\alpha)(1-\gamma),
\end{gathered}
$$

from expression (57)

$$
\alpha=\alpha_{1}^{*} / \beta,
$$

substituting expression (61) in expression (58) leads to

$$
\beta=\alpha_{1}^{*} /\left[\alpha_{1}^{*}+\alpha_{2}^{*}\right]
$$

substituting expression (62) in expression (61) leads to

$$
\alpha=\alpha_{1}^{*}+\alpha_{2}^{*}
$$

From expression (59)

$$
1-\alpha=\alpha_{3}^{*} / \gamma
$$

substituting expression (64) in expression (60) leads to

$$
\gamma=\alpha_{3}^{*} /\left[\alpha_{3}^{*}+\alpha_{4}^{*}\right]
$$

To ensure that $\beta \in[0,1]$ and $\gamma \in[0,1]$ for $\alpha \in[0,1]$, assume that

$$
\begin{aligned}
& \beta=\beta(\alpha)=\alpha^{x_{1}} \\
& \gamma=\gamma(\alpha)=\alpha^{x_{2}}
\end{aligned}
$$

Then, substituting expressions (62) and (63) in expression (66) he have

$$
\alpha_{1}^{*} /\left[\alpha_{1}^{*}+\alpha_{2}^{*}\right]=\left(\alpha_{1}^{*}+\alpha_{2}^{*}\right)^{x_{1}} .
$$

which leads to

$$
x_{1}=\left(\ln \left[\alpha_{1}^{*} /\left(\alpha_{1}^{*}+\alpha_{2}^{*}\right)\right]\right) /\left(\ln \left[\alpha_{1}^{*}+\alpha_{2}^{*}\right]\right) .
$$

Now, substituting expressions (63) and (65) in expression (67) he have

$$
\alpha_{3}^{*} /\left[\alpha_{3}^{*}+\alpha_{4}^{*}\right]=\left(\alpha_{1}^{*}+\alpha_{2}^{*}\right)^{x_{2}} .
$$

which leads to

$$
x_{2}=\left(\ln \left[\alpha_{3}^{*} /\left(\alpha_{3}^{*}+\alpha_{4}^{*}\right)\right]\right) /\left(\ln \left[\alpha_{1}^{*}+\alpha_{2}^{*}\right]\right) .
$$

Substituting expression (66) and (67)in expressions (57), (58), (59), and (60) leads to the fact that the share of reactors 1,2,3 and 4 in the risk of the PGPs are given by the following expressions

$$
\begin{gathered}
\alpha_{1}=\alpha \beta=\alpha^{x_{1}+1}, \\
\alpha_{2}=\alpha(1-\beta)=\alpha-\alpha^{x_{1}+1},
\end{gathered}
$$




$$
\begin{gathered}
\alpha_{3}=\alpha^{x_{2}}-\alpha^{x_{2}+1} . \\
\alpha_{4}=1-\alpha-\alpha^{x_{2}}+\alpha^{x_{2}+1} .
\end{gathered}
$$

Then, substituting expressions (70), (71), (72), and (73) into expression (56) leads to risk of the PGP:

$$
\sigma_{Y}^{2}=\left[\alpha^{x_{1}+1}\right]^{2} \sigma_{1}^{2}+\left[\alpha-\alpha^{x_{1}+1}\right]^{2} \sigma_{2}^{2}+\left[\alpha^{x_{2}}-\alpha^{x_{2}+1}\right]^{2} \sigma_{3}^{2}+\left[1-\alpha-\alpha^{x_{2}}+\alpha^{x_{2}+1}\right]^{2} \sigma_{4}^{2} .
$$

From expressions (57) and (70), the PGP with lowest risk is given when

$$
\alpha=\left[\alpha_{1}^{*}\right]^{\frac{1}{1+x_{1}}} .
$$

Now we need to find the portfolio with the greatest expected return. The expected return of the PGP is given by

$$
\mu_{Y}=\alpha_{1} \mu_{1}+\alpha_{2} \mu_{2}+\alpha_{3} \mu_{3}+\alpha_{4} \mu_{4}
$$

substituting expressions (70), (71), (72), and (73) into expression (76) leads to

$$
\mu_{Y}=\alpha^{1+x_{1}} \mu_{1}+\left(\alpha-\alpha^{1+x_{1}}\right) \mu_{2}+\left(\alpha^{x_{2}}-\alpha^{1+x_{2}}\right) \mu_{3}+\left(1-\alpha-\alpha^{x_{2}}+\alpha^{1+x_{2}}\right) \mu_{4},
$$

It is straightforward to obtain that

$$
d \mu_{Y} / d \alpha=\left(1+x_{1}\right) \alpha^{x_{1}}\left[\mu_{1}-\mu_{2}\right]+\left[x_{2} \alpha^{x_{2}-1}-\left(1+x_{2}\right) \alpha^{x_{2}}\right]\left[\mu_{3}-\mu_{4}\right]+\left[\mu_{2}-\mu_{4}\right] \geq 0,
$$

because of the assumption that $\mu_{1} \geq \mu_{2} \geq \mu_{3} \geq \mu_{4}$. Then, the PGP reaches its maximal expected return when $\alpha=1, \mu_{Y}=\mu_{1}$ and $\sigma_{Y}^{2}=\sigma_{1}^{2}$. However, there could be an alternative criterion to choose the maximal expected return of the PGP. For example, if the expected return of reactor 4 is the least risky, then, the criterion could be to choose $\alpha^{d f}$, such that $\sigma_{Y}^{2}\left(\alpha^{d f}\right)=\sigma_{4}^{2}$. Then, the maximal expected return of the PGP is given by $\mu\left(\alpha^{d f}\right)$. Then, the risk of the efficient portfolios is obtained by the shares of reactors 1,2 , 3 , and 4 , given by expressions (70), (71), (72), and (73) for $\left[\alpha_{1}^{*}\right]^{\frac{1}{1+x_{1}}} \leq \alpha \leq \alpha^{d f}$. As a consequence, the risk of the PGP in the EF is given by expression (74). The maximal expected return for every corresponding level of risk is given by expression (77). Note that $\mu\left(\alpha^{d f}\right) \leq \mu_{1}$ and $\sigma\left(\alpha^{d f}\right) \leq \sigma_{1}$.

[Proof of Proposition 8] The risk of the PGP of five countries is given by $\sigma_{Y}=\sqrt{\sum_{i=1}^{5} \alpha_{i}^{2} \sigma_{i}^{2}}$. Assume that expected return of country 2 is the least risky. If $\sigma_{i, j}=0$, for any values $i$ and $j$, from 1 to 5 , such that $i<j$. If $\alpha_{i} \in[0,1]$ for $i=1,2,3,4,5$, and $\sum_{i=1}^{5} \alpha_{i}=1$. For tractability, the majority of the proof uses the variance of the $\mathrm{PGP}$ instead of its $\mathrm{SD}$.

Proof of a) We need to find the weight given to countries $1,2,3,4$, and 5 , given by $\left(\alpha_{1}, \alpha_{2}, \alpha_{3}, \alpha_{4}, \alpha_{5}\right)$, which ensures the minimum risk of the PGP. For tractability, take $\alpha_{5}=1-\alpha_{1}-\alpha_{2}-\alpha_{3}-\alpha_{4}$. Then, the risk of the PGP is given by $\sigma_{Y}^{2}=\alpha_{1}^{2} \sigma_{1}^{2}+\alpha_{2}^{2} \sigma_{2}^{2}+\alpha_{3}^{2} \sigma_{3}^{2}+\alpha_{4}^{2} \sigma_{4}^{2}+\left(1-\alpha_{1}-\alpha_{2}-\alpha_{3}-\alpha_{4}\right)^{2} \sigma_{5}^{2}$. First, we find the critical point of the PGP. The FOC are as follows:

$$
\begin{aligned}
& \partial \sigma_{Y}^{2} / \partial \alpha_{1}=2 \alpha_{1} \sigma_{1}^{2}+2\left(1-\alpha_{1}-\alpha_{2}-\alpha_{3}-\alpha_{4}\right)(-1) \sigma_{5}^{2}=0, \\
& \partial \sigma_{Y}^{2} / \partial \alpha_{2}=2 \alpha_{2} \sigma_{2}^{2}+2\left(1-\alpha_{1}-\alpha_{2}-\alpha_{3}-\alpha_{4}\right)(-1) \sigma_{5}^{2}=0, \\
& \partial \sigma_{Y}^{2} / \partial \alpha_{3}=2 \alpha_{3} \sigma_{3}^{2}+2\left(1-\alpha_{1}-\alpha_{2}-\alpha_{3}-\alpha_{4}\right)(-1) \sigma_{5}^{2}=0, \\
& \partial \sigma_{Y}^{2} / \partial \alpha_{4}=2 \alpha_{3} \sigma_{3}^{2}+2\left(1-\alpha_{1}-\alpha_{2}-\alpha_{3}-\alpha_{4}\right)(-1) \sigma_{5}^{2}=0,
\end{aligned}
$$

from expression (78), we have

$$
\alpha_{1}\left[\sigma_{1}^{2}+\sigma_{5}^{2}\right]+\alpha_{2} \sigma_{5}^{2}+\alpha_{3} \sigma_{5}^{2}+\alpha_{4} \sigma_{5}^{2}=\sigma_{5}^{2}
$$

from expression (79), we have

$$
\alpha_{1} \sigma_{5}^{2}+\alpha_{2}\left[\sigma_{2}^{2}+\sigma_{5}^{2}\right]+\alpha_{3} \sigma_{5}^{2}+\alpha_{4} \sigma_{5}^{2}=\sigma_{5}^{2}
$$


from expression (80), we have

$$
\alpha_{1} \sigma_{5}^{2}+\alpha_{2} \sigma_{5}^{2}+\alpha_{3}\left[\sigma_{3}^{2}+\sigma_{5}^{2}\right]+\alpha_{4} \sigma_{5}^{2}=\sigma_{5}^{2}
$$

from expression (81), we have

$$
\alpha_{1} \sigma_{5}^{2}+\alpha_{2} \sigma_{5}^{2}++\alpha_{3} \sigma_{5}^{2}+\alpha_{4}\left[\sigma_{4}^{2}+\sigma_{5}^{2}\right]=\sigma_{5}^{2} .
$$

Expressions (82), (83), (84), and (85) lead to the following system of equations

$$
\left[\begin{array}{cccc}
\sigma_{1}^{2}+\sigma_{5}^{2} & \sigma_{5}^{2} & \sigma_{5}^{2} & \sigma_{5}^{2} \\
\sigma_{5}^{2} & \sigma_{2}^{2}+\sigma_{5}^{2} & \sigma_{5}^{2} & \sigma_{5}^{2} \\
\sigma_{5}^{2} & \sigma_{5}^{2} & \sigma_{3}^{2}+\sigma_{5}^{2} & \sigma_{5}^{2} \\
\sigma_{5}^{2} & \sigma_{5}^{2} & \sigma_{5}^{2} & \sigma_{4}^{2}+\sigma_{5}^{2}
\end{array}\right]\left[\begin{array}{c}
\alpha_{1} \\
\alpha_{2} \\
\alpha_{3} \\
\alpha_{4}
\end{array}\right]=\left[\begin{array}{c}
\sigma_{5}^{2} \\
\sigma_{5}^{2} \\
\sigma_{5}^{2} \\
\sigma_{5}^{2}
\end{array}\right]
$$

Calculating the inverse of matrix $A_{5}=\left[\begin{array}{cccc}\sigma_{1}^{2}+\sigma_{5}^{2} & \sigma_{5}^{2} & \sigma_{5}^{2} & \sigma_{5}^{2} \\ \sigma_{5}^{2} & \sigma_{2}^{2}+\sigma_{5}^{2} & \sigma_{5}^{2} & \sigma_{5}^{2} \\ \sigma_{5}^{2} & \sigma_{5}^{2} & \sigma_{3}^{2}+\sigma_{5}^{2} & \sigma_{5}^{2} \\ \sigma_{5}^{2} & \sigma_{5}^{2} & \sigma_{5}^{2} & \sigma_{4}^{2}+\sigma_{5}^{2}\end{array}\right]$ we end up with

$$
\left[\begin{array}{c}
\alpha_{1}^{*} \\
\alpha_{2}^{*} \\
\alpha_{3}^{*} \\
\alpha_{4}^{*}
\end{array}\right]=\frac{1}{\left|A_{5}\right|}\left[\begin{array}{cccc}
{\left[\begin{array}{c}
\sigma_{2}^{2} \sigma_{3}^{2} \sigma_{4}^{2}+ \\
\sigma_{2}^{2} \sigma_{3}^{2} \sigma_{5}^{2}+ \\
\sigma_{2}^{2} \sigma_{4}^{2} \sigma_{5}^{2}+ \\
\sigma_{3}^{2} \sigma_{4}^{2} \sigma_{5}^{2}
\end{array}\right]} & -\sigma_{3}^{2} \sigma_{4}^{2} \sigma_{5}^{2} & -\sigma_{2}^{2} \sigma_{4}^{2} \sigma_{5}^{2} & -\sigma_{2}^{2} \sigma_{3}^{2} \sigma_{5}^{2} \\
-\sigma_{3}^{2} \sigma_{4}^{2} \sigma_{5}^{2} & {\left[\begin{array}{c}
\sigma_{1}^{2} \sigma_{3}^{2} \sigma_{4}^{2}+ \\
\sigma_{1}^{2} \sigma_{3}^{2} \sigma_{5}^{2}+ \\
\sigma_{1}^{2} \sigma_{4}^{2} \sigma_{5}^{2}+ \\
\sigma_{3}^{2} \sigma_{4}^{2} \sigma_{5}^{2}
\end{array}\right]} & -\sigma_{1}^{2} \sigma_{4}^{2} \sigma_{5}^{2} & -\sigma_{1}^{2} \sigma_{3}^{2} \sigma_{5}^{2} \\
& & \\
-\sigma_{2}^{2} \sigma_{4}^{2} \sigma_{5}^{2} & -\sigma_{1}^{2} \sigma_{4}^{2} \sigma_{5}^{2} & {\left[\begin{array}{c}
\sigma_{1}^{2} \sigma_{2}^{2} \sigma_{4}^{2}+ \\
\sigma_{1}^{2} \sigma_{2}^{2} \sigma_{5}^{2}+ \\
\sigma_{1}^{2} \sigma_{4}^{2} \sigma_{5}^{2}+ \\
\sigma_{2}^{2} \sigma_{4}^{2} \sigma_{5}^{2}
\end{array}\right]} & -\sigma_{1}^{2} \sigma_{2}^{2} \sigma_{5}^{2} \\
& & \\
& & -\sigma_{1}^{2} \sigma_{2}^{2} \sigma_{5}^{2} & {\left[\begin{array}{c}
\sigma_{1}^{2} \sigma_{2}^{2} \sigma_{3}^{2}+ \\
\sigma_{1}^{2} \sigma_{2}^{2} \sigma_{5}^{2}+ \\
\sigma_{1}^{2} \sigma_{3}^{2} \sigma_{5}^{2}+ \\
\sigma_{2}^{2} \sigma_{3}^{2} \sigma_{5}^{2}
\end{array}\right]}
\end{array}\right]\left[\begin{array}{c}
\sigma_{5}^{2} \\
\sigma_{5}^{2} \\
\sigma_{5}^{2} \\
\sigma_{5}^{2}
\end{array}\right]
$$

where $\left|A_{5}\right|=\sigma_{1}^{2} \sigma_{2}^{2} \sigma_{3}^{2} \sigma_{4}^{2}+\sigma_{1}^{2} \sigma_{2}^{2} \sigma_{3}^{2} \sigma_{5}^{2}+\sigma_{1}^{2} \sigma_{2}^{2} \sigma_{4}^{2} \sigma_{5}^{2}+\sigma_{1}^{2} \sigma_{3}^{2} \sigma_{4}^{2} \sigma_{5}^{2}+\sigma_{2}^{2} \sigma_{3}^{2} \sigma_{4}^{2} \sigma_{5}^{2}$. The solution of the system of equations is

$$
\left[\begin{array}{c}
\alpha_{1}^{*} \\
\alpha_{2}^{*} \\
\alpha_{3}^{*} \\
\alpha_{4}^{*}
\end{array}\right]=\frac{1}{\left|A_{5}\right|}\left[\begin{array}{c}
\sigma_{2}^{2} \sigma_{3}^{2} \sigma_{4}^{2} \sigma_{5}^{2} \\
\sigma_{1}^{2} \sigma_{3}^{2} \sigma_{4}^{2} \sigma_{5}^{2} \\
\sigma_{1}^{2} \sigma_{2}^{2} \sigma_{4}^{2} \sigma_{5}^{2} \\
\sigma_{1}^{2} \sigma_{2}^{2} \sigma_{3}^{2} \sigma_{5}^{2}
\end{array}\right]
$$

Then, $\alpha_{5}^{*}=1-\alpha_{1}^{*}-\alpha_{2}^{*}-\alpha_{3}^{*}-\alpha_{4}^{*}=\sigma_{1}^{2} \sigma_{2}^{2} \sigma_{3}^{2} \sigma_{4}^{2} /\left|A_{5}\right|$. The critical point of the risk of the PGP is

$$
\left(\begin{array}{c}
\alpha_{1}^{*} \\
\alpha_{2}^{*} \\
\alpha_{3}^{*} \\
\alpha_{4}^{*} \\
\alpha_{5}^{*}
\end{array}\right)=\frac{1}{\left|A_{5}\right|}\left(\begin{array}{c}
\sigma_{2}^{2} \sigma_{3}^{2} \sigma_{4}^{2} \sigma_{5}^{2} \\
\sigma_{1}^{2} \sigma_{3}^{2} \sigma_{4}^{2} \sigma_{5}^{2} \\
\sigma_{1}^{2} \sigma_{2}^{2} \sigma_{4}^{2} \sigma_{5}^{2} \\
\sigma_{1}^{2} \sigma_{2}^{2} \sigma_{3}^{2} \sigma_{5}^{2} \\
\sigma_{1}^{2} \sigma_{2}^{2} \sigma_{3}^{2} \sigma_{4}^{2}
\end{array}\right)
$$

To verify that the risk of the PGP, $\sigma_{Y}^{2}$, has a minimum at critical point $\left(\alpha_{1}^{*}, \alpha_{2}^{*}, \alpha_{3}^{*}, \alpha_{4}^{*}, \alpha_{5}^{*}\right)$, we need the 
SOC. The Hessian matrix is given as follows:

$$
H=2\left[\begin{array}{cccc}
\sigma_{1}^{2}+\sigma_{5}^{2} & \sigma_{5}^{2} & \sigma_{5}^{2} & \sigma_{5}^{2} \\
\sigma_{5}^{2} & \sigma_{2}^{2}+\sigma_{5}^{2} & \sigma_{5}^{2} & \sigma_{5}^{2} \\
\sigma_{5}^{2} & \sigma_{5}^{2} & \sigma_{3}^{2}+\sigma_{5}^{2} & \sigma_{5}^{2} \\
\sigma_{5}^{2} & \sigma_{5}^{2} & \sigma_{5}^{2} & \sigma_{4}^{2}+\sigma_{5}^{2}
\end{array}\right]
$$

Following the criteria of the leading principal minors of the Hessian matrix, we have

$$
\begin{gathered}
H_{1}=2\left(\sigma_{1}^{2}+\sigma_{5}^{2}\right)>0, \\
H_{2}=2\left|\begin{array}{cc}
\sigma_{1}^{2}+\sigma_{5}^{2} & \sigma_{5}^{2} \\
\sigma_{5}^{2} & \sigma_{2}^{2}+\sigma_{5}^{2}
\end{array}\right|=2\left(\sigma_{1}^{2} \sigma_{2}^{2}+\sigma_{1}^{2} \sigma_{5}^{2}+\sigma_{2}^{2} \sigma_{5}^{2}\right)>0, \\
H_{3}=2\left|\begin{array}{ccc}
\sigma_{1}^{2}+\sigma_{5}^{2} & \sigma_{5}^{2} & \sigma_{5}^{2} \\
\sigma_{5}^{2} & \sigma_{2}^{2}+\sigma_{5}^{2} & \sigma_{5}^{2} \\
\sigma_{5}^{2} & \sigma_{5}^{2} & \sigma_{3}^{2}+\sigma_{5}^{2}
\end{array}\right|=2\left(\sigma_{1}^{2} \sigma_{2}^{2} \sigma_{3}^{2}+\sigma_{1}^{2} \sigma_{2}^{2} \sigma_{5}^{2}+\sigma_{1}^{2} \sigma_{3}^{2} \sigma_{5}^{2}+\sigma_{2}^{2} \sigma_{3}^{2} \sigma_{5}^{2}\right)>0, \\
H_{4}=2\left|A_{5}\right|>0 .
\end{gathered}
$$

The four leading principal minors of the Hessian matrix are positive for any $\left(\alpha_{1}, \alpha_{2}, \alpha_{3}, \alpha_{4}, \alpha_{5}\right)$. Thus, the risk of the PGP is a convex function of the weight received by the five countries $\left(\alpha_{1}, \alpha_{2}, \alpha_{3}, \alpha_{4}, \alpha_{5}\right)$. As a consequence, the risk of the PGP, $\sigma_{Y}^{2}$, has a global minimum when the weights received by the five countries are given by expression (89).

Proof of $\mathbf{b})$ Then, the minimal risk of the PGP, $\sigma_{Y}^{* 2}$, is given by

$$
\begin{gathered}
\sigma_{Y}^{* 2}=\left(1 /\left|A_{5}\right|^{2}\right)\left[\left(\sigma_{2}^{2} \sigma_{3}^{2} \sigma_{4}^{2} \sigma_{5}^{2}\right)^{2} \sigma_{1}^{2}+\left(\sigma_{1}^{2} \sigma_{3}^{2} \sigma_{4}^{2} \sigma_{5}^{2}\right)^{2} \sigma_{2}^{2}+\right. \\
\left.\left(\sigma_{1}^{2} \sigma_{2}^{2} \sigma_{4}^{2} \sigma_{5}^{2}\right)^{2} \sigma_{3}^{2}+\left(\sigma_{1}^{2} \sigma_{2}^{2} \sigma_{3}^{2} \sigma_{5}^{2}\right)^{2} \sigma_{4}^{2}+\left(\sigma_{1}^{2} \sigma_{2}^{2} \sigma_{3}^{2} \sigma_{4}^{2}\right)^{2} \sigma_{5}^{2}\right] \\
\sigma_{Y}^{* 2}=\left(\sigma_{1}^{2} \sigma_{2}^{2} \sigma_{3}^{2} \sigma_{4}^{2} \sigma_{5}^{2} /\left|A_{5}\right|^{2}\right)\left[\sigma_{2}^{2} \sigma_{3}^{2} \sigma_{4}^{2} \sigma_{5}^{2}+\sigma_{1}^{2} \sigma_{3}^{2} \sigma_{4}^{2} \sigma_{5}^{2}+\sigma_{1}^{2} \sigma_{2}^{2} \sigma_{4}^{2} \sigma_{5}^{2}+\sigma_{1}^{2} \sigma_{2}^{2} \sigma_{3}^{2} \sigma_{5}^{2}+\sigma_{1}^{2} \sigma_{2}^{2} \sigma_{3}^{2} \sigma_{4}^{2}\right], \\
\sigma_{Y}^{* 2}=\left(\sigma_{1}^{2} \sigma_{2}^{2} \sigma_{3}^{2} \sigma_{4}^{2} \sigma_{5}^{2} /\left|A_{5}\right|\right)=\alpha_{3}^{*} \sigma_{3}^{2}<\sigma_{3}^{2},
\end{gathered}
$$

Then

$$
\sigma_{Y}^{*}<\sigma_{3} .
$$

The minimal risk of the PGP is lower than the risk of the least risky country.

[Proof of Proposition 9] Let $\sigma_{Y}=\sqrt{\sum_{i=1}^{5} \alpha_{i}^{2} \sigma_{i}^{2}}$ be risk of the PGP of five countries. From Proposition 10 , we know that the risk of the PGP, $\sigma_{Y}$, reaches its global minimum at point $\left(\alpha_{1}^{*}, \alpha_{2}^{*}, \alpha_{3}^{*}, \alpha_{4}^{*}, \alpha_{5}^{*}\right)$. Assume that $\mu_{1} \geq \mu_{2} \geq \mu_{3} \geq \mu_{4} \geq \mu_{5}$. To obtain the parametric formulation of the EF, we express the risk of the PGP as follows:

$$
\sigma_{Y}^{2}=\alpha^{2}\left(\eta^{2}\left[\beta^{2} \sigma_{1}^{2}+(1-\beta)^{2} \sigma_{2}^{2}\right]+(1-\eta)^{2}\left[\gamma^{2} \sigma_{3}^{2}+(1-\gamma)^{2} \sigma_{4}^{2}\right]\right)+(1-\alpha)^{2} \sigma_{5}^{2}, \sigma_{Y}^{2}=\alpha^{2} \eta^{2} \beta^{2} \sigma_{1}^{2}+\alpha^{2} \eta^{2}(1-\beta)^{2} \sigma_{2}^{2}+\alpha^{2}(1
$$

for $\alpha, \eta, \beta, \gamma \in[0,1]$. Note that when $\alpha=\beta=\eta=1$, then $\sigma_{Y}^{2}=\sigma_{1}^{2}$, the risk of the PGP equals the risk of country 1 . This fact implies that country 1 , which has the greatest expected return, receives a share of $100 \%$. On the other hand, when $\alpha=0$, then $\sigma_{Y}^{2}=\sigma_{5}^{2}$, the risk of the PGP equals the risk of country 5 . Then, country 5 , which has the least expected return, receives a share of $100 \%$. Then, this formulation of the risk of the PGP allows to have portfolios that assign a share of $100 \%$ to the countries with the greatest and least expected return. We need to be sure that expression (92) allows to reach the point $\left(\alpha_{1}^{*}, \alpha_{2}^{*}, \alpha_{3}^{*}, \alpha_{4}^{*}, \alpha_{5}^{*}\right)$, where $\sigma_{Y}$ reaches its global minimum. Then, it should hold that

$$
\begin{gathered}
\alpha_{1}^{*}=\alpha \eta \beta, \\
\alpha_{2}^{*}=\alpha \eta(1-\beta), \\
\alpha_{3}^{*}=\alpha(1-\eta) \gamma,
\end{gathered}
$$




$$
\begin{gathered}
\alpha_{4}^{*}=\alpha(1-\eta)(1-\gamma), \\
\alpha_{5}^{*}=(1-\alpha),
\end{gathered}
$$

from expression (93)

$$
\alpha \eta=\alpha_{1}^{*} / \beta
$$

substituting expression (98) in expression (94) leads to

$$
\beta=\alpha_{1}^{*} /\left[\alpha_{1}^{*}+\alpha_{2}^{*}\right]
$$

substituting expression (99) in expression (98) leads to

$$
\alpha \eta=\alpha_{1}^{*}+\alpha_{2}^{*} .
$$

From expression (97)

$$
\alpha=1-\alpha_{5}^{*},
$$

substituting expression (101) into expression (100) leads to

$$
\eta=\left[\alpha_{1}^{*}+\alpha_{2}^{*}\right] /\left[1-\alpha_{5}^{*}\right]
$$

From expression (95)

$$
\alpha(1-\eta)=\alpha_{3}^{*} / \gamma,
$$

substituting expression (103) in expression (96) leads to

$$
\gamma=\alpha_{3}^{*} /\left[\alpha_{3}^{*}+\alpha_{4}^{*}\right]
$$

To ensure that $\beta \in[0,1], \eta \in[0,1]$ and $\gamma \in[0,1]$ for $\alpha \in[0,1]$, assume that

$$
\begin{aligned}
& \beta=\beta(\alpha)=\alpha^{x_{1}} \\
& \eta=\eta(\alpha)=\alpha^{x_{2}} \\
& \gamma=\gamma(\alpha)=\alpha^{x_{3}}
\end{aligned}
$$

Then, substituting expressions (99) and (101) into expression (105) he have

$$
\alpha_{1}^{*} /\left[\alpha_{1}^{*}+\alpha_{2}^{*}\right]=\left(1-\alpha_{5}^{*}\right)^{x_{1}}
$$

which leads to

$$
x_{1}=\left[\ln \left(\alpha_{1}^{*} /\left[\alpha_{1}^{*}+\alpha_{2}^{*}\right]\right)\right] /\left(\ln \left[1-\alpha_{5}^{*}\right]\right) .
$$

Substituting expressions (101) and (104) in expression (106) he have

$$
\left[\alpha_{1}^{*}+\alpha_{2}^{*}\right] /\left[1-\alpha_{5}^{*}\right]=\left(1-\alpha_{5}^{*}\right)^{x_{2}} .
$$

which leads to

$$
x_{2}=\left[\ln \left(\left[\alpha_{1}^{*}+\alpha_{2}^{*}\right] /\left[1-\alpha_{5}^{*}\right]\right)\right] /\left(\ln \left[1-\alpha_{5}^{*}\right]\right) .
$$

Substituting expressions (101) and (102) in expression (107) he have

$$
\alpha_{3}^{*} /\left[\alpha_{3}^{*}+\alpha_{4}^{*}\right]=\left(1-\alpha_{5}^{*}\right)^{x_{3}} .
$$

which leads to

$$
x_{3}=\left[\ln \left(\alpha_{3}^{*} /\left[\alpha_{3}^{*}+\alpha_{4}^{*}\right]\right)\right] /\left(\ln \left[1-\alpha_{5}^{*}\right]\right) .
$$

Substituting expression (105), (106) and (107) into expressions (93), (94), (95), (96), and (97) leads to the fact that the weights received by countries $1,2,3,4$ and 5 in the risk of the PGPs are given by the following expressions,

$$
\alpha_{1}=\alpha \eta \beta=\alpha^{1+x_{1}+x_{2}}
$$




$$
\begin{gathered}
\alpha_{2}=\alpha \eta(1-\beta)=\alpha^{1+x_{2}}-\alpha^{1+x_{1}+x_{2}} \\
\alpha_{3}=\alpha(1-\eta) \gamma=\alpha^{1+x_{3}}-\alpha^{1+x_{2}+x_{3}} \\
\alpha_{4}=\alpha(1-\eta)(1-\gamma)=\alpha-\alpha^{1+x_{2}}-\alpha^{1+x_{3}}+\alpha^{1+x_{2}+x_{3}} \\
\alpha_{5}=1-\alpha .
\end{gathered}
$$

Then, substituting expressions (111), (112), (113), (114), and (115) in expression (92) leads to the risk of the PGP:

$$
\sigma_{Y}^{2}=\left[\alpha^{1+x_{1}+x_{2}}\right]^{2} \sigma_{1}^{2}+\left[\alpha^{1+x_{2}}-\alpha^{1+x_{1}+x_{2}}\right]^{2} \sigma_{2}^{2}+\left[\alpha^{1+x_{3}}-\alpha^{1+x_{2}+x_{3}}\right]^{2} \sigma_{3}^{2}+\left[\alpha-\alpha^{1+x_{2}}-\alpha^{1+x_{3}}+\alpha^{1+x_{2}+x_{3}}\right]^{2} \sigma_{4}^{2}+[1-\alpha]^{2}
$$

From expressions (93) and (111), the PGP with lowest risk is given when

$$
\alpha=\left[\alpha_{1}^{*}\right]^{\frac{1}{1+x_{1}+x_{2}}} .
$$

Now we need to find the portfolio with the corresponding greatest expected return. The expected return of the PGP is given by

$$
\mu_{Y}=\alpha_{1} \mu_{1}+\alpha_{2} \mu_{2}+\alpha_{3} \mu_{3}+\alpha_{4} \mu_{4}+\alpha_{5} \mu_{5},
$$

substituting expressions (111), (112), (113), (114), and (115) in expression (118) leads to

$$
\mu_{Y}=\left(\alpha^{1+x_{1}+x_{2}}\right) \mu_{1}+\left(\alpha^{1+x_{2}}-\alpha^{1+x_{1}+x_{2}}\right) \mu_{2}+\left(\alpha^{1+x_{3}}-\alpha^{1+x_{2}+x_{3}}\right) \mu_{3}+\left(\alpha-\alpha^{1+x_{2}}-\alpha^{1+x_{3}}+\alpha^{1+x_{2}+x_{3}}\right) \mu_{4}+(1-\alpha) \mu_{5}
$$

It is straightforward to obtain that

$$
d \mu_{Y} / d \alpha=\left(1+x_{1}+x_{2}\right) \alpha^{x_{1}+x_{2}}\left[\mu_{1}-\mu_{2}\right]+\left(1+x_{2}\right) \alpha^{x_{2}}\left[\mu_{2}-\mu_{4}\right]+\left[\left(1+x_{3}\right) \alpha^{x_{3}}-\left(1+x_{2}+x_{3}\right) \alpha^{x_{2}+x_{3}}\right]\left[\mu_{3}-\mu_{4}\right]+\left[\mu_{4}-\mu_{5}\right.
$$

because of the assumption that $\mu_{1} \geq \mu_{2} \geq \mu_{3} \geq \mu_{4} \geq \mu_{5}$. Then, the PGP reaches its maximal expected return when $\alpha=1, \mu_{Y}=\mu_{1}$ and $\sigma_{Y}^{2}=\sigma_{1}^{2}$. However, there could be an alternative criterion to choose the maximum expected return of the PGP. For example, if the expected return of country 3 is the least risky, then, the criterion could be to choose $\alpha^{d f}$, so that $\sigma_{Y}^{2}\left(\alpha^{d f}\right)=\sigma_{3}^{2}$. Then, the maximal expected return of the PGP is given by $\mu\left(\alpha^{d f}\right)$. The risk of the efficient portfolios is obtained by the weights received by the wind production of countries 1, 2, 3, 4, and 5, given by expressions (111), (112), (113), (114), and (115) for $\left[\alpha_{1}^{*}\right]^{\frac{1}{1+x_{1}+x_{2}}} \leq \alpha \leq \alpha^{d f}$. As a consequence, the risk of the PGP in the EF is given by expression (116). The maximal expected return for every corresponding level of risk is given by expression (119). Note that $\mu\left(\alpha^{d f}\right) \leq \mu_{1}$ and $\sigma\left(\alpha^{d f}\right) \leq \sigma_{1}$. 